\title{
25 Research Square \\ Bcl-2-associated Transcription Factor 1 \\ Phosphorylation Mediated DNA Damage Response in Gastric Cancer
}

Jia Liu ( $\square$ liujiajzc@mail.sdu.edu.cn )

Shandong University Cheeloo College of Medicine

Jingyi Li

Zhengzhou University

Zhao Sun

Zhengzhou University

Yangmiao Duan

Shandong University Cheeloo College of Medicine

Fengqin Wang

Shandong University Cheeloo College of Medicine

Guangwei Wei

Shandong University Cheeloo College of Medicine

Jing-Hua Yang

Zhengzhou University First Affiliated Hospital

\section{Research Article}

Keywords: Phosphoproteomics, BCLAF1, Ser290, DNA damage response, Gastric cancer

Posted Date: April 20th, 2021

DOl: https://doi.org/10.21203/rs.3.rs-431350/v1

License: (9) (i) This work is licensed under a Creative Commons Attribution 4.0 International License.

Read Full License

Version of Record: A version of this preprint was published at Journal of Translational Medicine on August 9th, 2021. See the published version at https://doi.org/10.1186/s12967-021-03004-z. 


\section{Abstract}

Background: DNA damage response plays critical roles in tumor pathogenesis and radiotherapy resistance. Various cellular activities including the initiation and major steps DNA damage response are regulated by protein phosphorylation, whereas the molecular mechanism remains largely unknown.

Methods: A quick label-free phosphoproteomics using high-resolution mass spectrometry and an open search approach was applied to paired tumor and adjacent tissues from five patients with gastric cancer. The dysregulated phosphoproteins were identified and their associated-pathways analyzed using Gene Set Enrichment Analysis (GSEA). The mostly regulated phosphoproteins and their potential functions were validated by the specific antibodies against the phosphorylation sites. Specific protein phosphorylation was further analyzed by functional and clinical approaches.

Results: 832 gastric cancer-associated unique phosphorylated sites were identified, among which 25 were up- and 52 down-regulated. Markedly, the dysregulated phosphoproteins were primarily enriched in DNAdamage-response-associated pathways. Particularly, the phosphorylation of Bcl-2-associated transcription factor 1 (BCLAF1) at Ser290 was significantly upregulated in tumor. The upregulation of BCLAF1 Ser290 phosphorylation (pBCLAF1(Ser290)) in tumor was confirmed by tissue microarray studies and further indicated in association with poor prognosis of gastric cancer patients. Eliminating the phosphorylation of BCLAF1 at Ser290 suppressed gastric cancer (GC) cell proliferation. Upregulation of pBCLAF1(Ser290) was found in association with irradiation-induced $\mathrm{y}-\mathrm{H} 2 \mathrm{AX}$ expression in the nucleus, leading to an increased DNA damage repair response, and a marked inhibition of irradiation-induced cancer cell apoptosis.

Conclusions: The phosphorylation of BCLAF1 at Ser290 is involved in the regulation of DNA damage response, indicating an important target for the resistance of radiotherapy.

\section{Background}

GC is one of the most aggressive and therapy-resistant cancers [1]. In China, approximately 405,000 new cases and 325,000 deaths from GC have been reported, making it the second most prevalent disease and the third in cancer-related deaths [2]. Early onset GC is difficult to diagnose due to the histological and genetic heterogeneity of the disease [3]. GC patients are often diagnosed after the disease has progressed to the advanced stage where the long term outlook is very poor and the 5-year survival rate is only $10 \%-20 \%$ [4]. Current treatment strategies for GC include surgical resection, chemotherapy and radiation therapy, however the effects are limited $[5,6]$. In recent years, the development of molecular targeted therapy has led to a revolutionary breakthrough and become the hope of cancer treatment. The protein phosphorylation is a critical post-translational modification and therapeutic target in regulating different biological processes [7-9] important for diagnosis, prognosis and treatment of diseases. Thus, a better understanding of GC phosphoproteomics can improve early diagnostic screening and provide effective intervention targets. 
Mass spectrometry-based shotgun proteomics has become the leading technology to investigate alteration or modification of proteins [10-12]. It was applied to identify the phosphoproteins in GC, leading to the identification of 162 phosphorylation sites on 49 nonredundant proteins [13]. Notably, Bcl-2associated transcription factor 1 (BCLAF1), a death-promoting transcriptional repressor highly expressed in a variety types of cancer [14-17], is shown to be phosphorylated at multiple positions. BCLAF1 is involved in a wide range of biological processes including apoptosis, transcriptional regulation and DNA damage repair [18-21]. The BCLAF1 protein contains homologies to the basic zipper and Myb DNAbinding domain and can bind to DNA [22]. Several studies have shown that BCLAF1 plays an important role in DNA damage repair (DDR) [19, 23]. BCLAF1 promotes the transcription of TP53 gene by interaction with PKC $\delta$ in response to DNA damage and interacts with $\mathrm{Y}-\mathrm{H} 2 \mathrm{AX}$ upon ionizing radiation (IR) [24]. BCLAF1 also induces cisplatin resistance in lung cancer cells by regulating DNA damage repair [18]. However, it remained to be determined how BCLAF1 phosphorylation regulates DNA damage response.

In recent years, high-resolution technology in mass spectrometry and better open search algorithms are developed. On this basis, we have developed a quick label-free phosphoproteomics workflow and identified 832 unique phosphorylated sites over 382 proteins from GC, particularly BCLAF1 at Ser290. Bioinformatics analysis showed that the upregulated phosphoproteins were enriched in association with the molecular functions in relevant to DNA damage repair, tumorigenesis and therapy resistance. To address the underlying mechanism, GC cells stably overexpressing BCLAF1 and its mutants were established and their effects on DNA damage repair upon exposure to IR were determined. It was further validated by the clinical data that BCLAF1 Ser290 phosphorylation was upregulated in association with poor prognosis of GC patients.

\section{Methods}

\section{Clinical patient samples}

This study was conducted after approval by the Ethic Committee of Shandong University, and informed written consent was obtained from all patients. All of the gastric carcinoma tissues and paired normal tissues were obtained from five patients treated in the First Affiliated Hospital of the Fourth Military Medical University. The protocol for proteomics analysis of the samples were approved by the Institutional Research Ethics Review Boards. To avoid necrotic and adjacent non-tumor tissues inclusion, cancer tissues were taken from the core area of tumor. Adjacent tissues were taken from non-tumor regions at least $3 \mathrm{~cm}$ away from the core area of tumor. All tissues were verified by two pathologists and frozen immediately in liquid nitrogen and stored at $-80^{\circ} \mathrm{C}$.

\section{Cell lines and cell culture}

The human GC cell lines AGS and HGC-27 (HGC) used in this study were obtained from ATCC. HGC cells were cultured in RPMI-1640 medium containing with 10\% fetal bovine serum (FBS) and 1\% penicillin/streptomycin. AGS cells were cultured in Dulbecco's modified Eagle's medium (DMEM) (Gibco) 
with $10 \%$ FBS and $1 \%$ penicillin/streptomycin (Gibco). All cells were cultured in a sterile incubator humidified atmosphere containing $5 \% \mathrm{CO}_{2}$ at $37^{\circ} \mathrm{C}$.

\section{LC-MS/MS sample preparation}

Proteins were extracted from five pairs of human gastric tumor and adjacent non-tumor sample using TPER buffer (Thermo Fisher Scientific) with protease inhibitor cocktail (Sigma-Aldrich) and phosphatase cocktail (Sigma-Aldrich). The protein concentrations of tissue lysates were quantified by Bicinchoninic Acid (BCA) Protein Assay kit (Pierce, thermo scientific, Germany). Triplicate lysates and bovine serum albumin (BSA) standards were added to a 96-well plate which contained $100 \mu \mathrm{L}$ of working reagent followed by incubation for $30 \mathrm{~min}$ at $37^{\circ} \mathrm{C}$. The absorbance was measured at $562 \mathrm{~nm}$ on a Microplate Reader (Thermo Fisher Scientific). The linear standard curve formula of protein quantification was established by drawing the relationship between the blank corrected BSA standard absorbance and the BSA standard concentration. The absorbance of the blank corrected sample was put into the linear standard curve formula to calculate the protein concentration of the sample. Equal amounts of the lysates were mixed with $8 \mathrm{M}$ urea (UA) in Microcon devices (YM-30, Millipore). Proteins were reduced using $10 \mathrm{mM}$ dithiothreitol and then alkylated using $50 \mathrm{mM}$ iodoacetamide (IAA) in the dark. In the digestion step, proteins were digested with trypsin (Promega) at a protein:trypsin ratio of 25:1 overnight at $37^{\circ} \mathrm{C}$. Peptides were desalted by ZipTip C18 pipette tips (Millipore), washed with $0.1 \%$ trifluoroacetic acid (TFA), and eluted with $50 \%$ methanol followed by dry in a SpeedVac for LC-MS/MS analysis.

\section{High pressure liquid chromatography}

The mixed peptides were separated by high pressure liquid chromatography (HPLC) on an EASY-nLC 1000 system (Thermo Scientific, San Jose, $\mathrm{CA}$ ). In brief, first set $2 \% \mathrm{CH}_{3} \mathrm{CN}$ in water, $\mathrm{NH}_{3} \mathrm{H}_{2} \mathrm{O}$ to adjust pH 10.0 as Phase $\mathrm{A}, 98 \% \mathrm{CH}_{3} \mathrm{CN}$ in water as Phase B. Peptides were dissolved in $80 \mu \mathrm{L}$ phase $\mathrm{A}$ and fractionated with a long C18 column (300 ' $\varnothing 0.075 \mathrm{~mm}, 3 \mu \mathrm{m}$; Reprosil, Germany) with a mobile phase gradient ( 0-3 min, 100\% phase A, 3-5 min, 100\% -70\% phase A, $5-45 \mathrm{~min}, 70 \%-30 \%$ phase $A, 45-55 \mathrm{~min}$, 30-5\% phase A, 55-60 min, 5\% phase A). Finally, 55 components were collected and dried in SpeedVac. Using $50 \% \mathrm{CH}_{3} \mathrm{CN}$ and $1 \%$ TFA in water to dissolve 55 components and merge them into 10 components which finally dried under vacuum in SpeedVac for subsequent mass spectrometry analysis.

\section{Mass spectrometry analysis}

The MS and MS/MS spectra were acquired by an LTQ-Orbitrap Elite mass spectrometer (Thermo Scientific, San Jose, CA) in data-dependent mode. The spray voltage was $2.1 \mathrm{kV}$ and the capillary temperature $275^{\circ} \mathrm{C}$. MS spectra were acquired in profile mode in the $\mathrm{m} / \mathrm{z}$ range of $350-1800$ at a resolution of 60,000 at $400 \mathrm{~m} / \mathrm{z}$. MS/MS fragmentation of the 30 most intense peaks was performed for every full MS scan in the collision-induced dissociation mode. Triple technical replicates were analyzed for each sample.

\section{Protein sequencing alignment and single amino acid polymorphisms analysis}


MS/MS spectra were searched against the human protein database using SEQUEST in Proteome Discover. Trypsin (full cleavage) was specified as cleavage enzyme allowing up to two missing cleavages. MS/MS spectra were searched with a maximum allowed deviation of $10 \mathrm{ppm}$ for the precursor mass and 0.6 Da for fragment masses. The oxidation of various amino acid residues including methionine was selected as dynamic phosphorylation (+79.9663 Da), and the false discovery rate (FDR) was $1 \%$. For phosphorylation analysis, an open search algorithm provided by Byonic and MODa was used. For clustering, mass shifts (delta masses) were divided into subgroups with $1 \mathrm{Da}$ intervals bounded by $n-0.5$ and $n+0.5 \mathrm{Da}(\mathrm{n}=-200$ to 1000$)$. Mass shifts in each mass window were analyzed by multivariate clustering using Gaussian mixture components. Clusters within each window were determined by the Bayesian Information Criterion (BIC). Next, clusters in each window were fitted individually with Gaussian regression to calculate the peak value (expected mass shifts), the SD, and the Goodness-of-Fit $\left(\mathrm{R}^{2}\right)$. Based on the given delta mass cluster which reflected the phosphorylation modifications, all the confident peptides were classified and normalized according to the spectral count.

\section{Protein-Protein Interaction (PPI) Network Analysis}

Using the STRING database (http://string-db.org/), PPI analysis for the phosphorylated differential proteins was conducted. The threshold for PPI analysis used the default values in the database.

\section{Gene Ontology (GO) Functional analysis}

The upregulated (above FC 2, p<0.05) and the downregulated (below FC 0.5, $p<0.05$ ) genes were sorted into different groups based on the fold change in gene expression. Each group was subjected to gene ontology (GO) functional annotation analysis under biological processes category, cell component and molecular function using DAVID Bioinformatics Resources (http://david.abcc.ncifcrf.gov/home.jsp) to determine significantly enriched genes. Pathways were identified from the Kyoto encyclopedia of genes and genomes (KEGG) database.

\section{Immunoprecipitation}

Cell lysate of HGC was carried out using RIPA Lysis Buffer (weak, Beyotime, China) and then incubated with $1 \mu \mathrm{g}$ of the BCLAF1 antibody overnight at $4^{\circ} \mathrm{C}$ on a rotating wheel. $30 \mu \mathrm{L}$ of protein A/G beads (MCE, China) were added to lysates and incubated on a rotator at $4^{\circ} \mathrm{C}$ for 2 hours. The beads were collected by a magnetic rack, and washed three times with ice-cold PBS-T (0.01\% Tween) buffer. Beads were incubated with loading buffer and boiled for 5 minutes followed by sodium dodecyl sulfatepolyacrylamide gel electrophoresis (SDS-PAGE) and western blot analysis.

\section{Protein in-gel digestion}

The SDS/PAGE gels were cut into small pieces, washed twice with $50 \mathrm{mM} \mathrm{NH}_{4} \mathrm{HCO}_{3}$ buffer at $4^{\circ} \mathrm{C}$ for $4 \mathrm{~h}$ and then dried with $100 \% \mathrm{CH}_{3} \mathrm{CN}$ for 10 min. Sequencing grade modified trypsin (Promega) at a concentration of $10 \mu \mathrm{g} / \mathrm{mL}$ in $50 \mathrm{mM} \mathrm{NH}_{4} \mathrm{HCO}_{3}$ buffer was added for overnight digestion at $37^{\circ} \mathrm{C}$. The 
peptides were extracted from the gel with $60 \% \mathrm{CH}_{3} \mathrm{CN}$ in $0.1 \%$ TFA for $30 \mathrm{~min}$. The extracted solution was dried under vacuum in SpeedVac for subsequent mass spectrometry analysis. The obtained data were submitted to Mascot software (Matrix Science) to search for phosphorylated residues on the BCLAF1 protein.

\section{Antibodies}

The specific antibody recognizing pBCLAF1(Ser290) was raised in rabbits against peptide coupling with PSQNS(p)PIH-KLH (corresponding to the residues 286-293 of human BCLAF1). The antibody was prepared and purified by ABclonal Biotech (China). Antibodies used in this study as follow: BCLAF1 (A300-608A) was purchased from Thermo Fisher Scientific, Inc. (USA); $Y$-H2AX (\#80312), Anti-rabbit IgG $(\mathrm{H}+\mathrm{L}$, Alexa Fluor 549 Conjugate, \#8889), Anti-mouse IgG (H+L, Alexa Fluor 488 Conjugate, \#4408), were purchased from Cell Signaling Technology, Inc. (USA); GAPDH (60004-1-Ig), Flag (F1804), GFP (F1804) were purchased from Proteintech, Ltd. (Shanghai, China).

\section{Human gastric Cancer Tissue Microarray, Immunohistochemistry, and Scoring}

Human GC tissue microarray (TMA) that consisted of the gastric tumor tissue specimens $(n=95)$ and adjacent non-normal tissue specimens $(n=85)$ were purchased from Outdo Biotech, Ltd. (Shanghai, China). The pBCLAF1(Ser290) antibody was used at a 1:100 dilution. The assignment of staining intensity score was based on the staining intensity (no intensity: 0 , weak intensity: $1+$, moderate intensity: $2+$, and strong intensity: $3+$ ) and positive-staining score was based on the percentage of positive-staining ( $0 \%$ positive: $0,1-25 \%$ positive: $1,26-50 \%$ positive: $2,51-75 \%$ positive: 3 , and $76-100 \%$ positive: 4 ) by three experienced pathologists. The final staining index was calculated using the formula: positivestaining score $\times$ staining intensity score. For data analysis, staining scores $<4$ were defined as low expression, and scores $>=4$ indicated high expression.

\section{Lentiviral plasmid construction and infection}

BCLAF1 Lentiviral short hairpin RNA (shRNA) and a negative control vector (NC) were purchased from GeneChem Co., Ltd. (Shanghai, China) and transduced into the HGC and AGS cell lines following the manufacturer's instructions. The Flag-tagged WT, S290D, and S290A overexpressed lentiviruses were amplified, purified from GeneChem Co., Ltd. (Shanghai, China). The cDNA was subcloned into the GV492 plasmid (Ubi-MCS-3FLAG-CBh-gcGFP-IRES-puromycin) (Genechem) and then co-transfected into HEK293T cells with the lentiviral genomic plasmids. Lentiviral particles were obtained by collecting supernatant using the kit for ultracentrifugation concentration and purification of lentiviral particles. Cells were cultured in 6-well plates until $60 \%$ confluent and infected with lentivirus particles at a $\mathrm{MOI}$ of 50 in the presence of $10 \mathrm{~g} / \mathrm{ml}$ polybrene for $48 \mathrm{~h}$. Stable cells were maintained in medium containing 0.5 $\mu \mathrm{g} / \mathrm{mL}$ of puromycin.

\section{MTT assay}


Three replicates of equal amounts of cells $\left(3 \times 10^{3} /\right.$ well) were seeded into 96 -well plates and incubated for various durations. The cells were incubated with $20 \mu \mathrm{L} 3-(4,5$-dimethylthiazol- 2-yl)-2, 5-

diphenyltetrazolium bromide (MTT, $5 \mathrm{mg} / \mathrm{ml}$ in PBS) for 4 hours at $37^{\circ} \mathrm{C}$. Then, $150 \mu \mathrm{L}$ dimethyl sulfoxide (DMSO) was added to the wells, and the optical density (OD) was detected at $490 \mathrm{~nm}$ by a microplate reader.

\section{Colony formation assay}

Exponentially growing cells $\left(5 \times 10^{2}\right)$ were seeded into 6 -well plates for $10-14$ days to form colonies. For the determination of colony formation, the cells were fixed in $4 \%$ polyformaldehyde, stained with $1 \%$ crystal violet. Colonies of at least 50 cells were counted. The mean \pm SD from three independent experiments was determined.

\section{5-Ethynyl-2'-deoxyuridine proliferation assay}

The 5-ethynyl-2》-deoxyuridine (EdU) assay was performed with an EdU Kit (Byotime, China). Cells were seeded onto 24-well plates and cultured in $0.5 \mathrm{~mL}$ medium for 24 hours, and then added $0.5 \mathrm{~mL}$ of $10 \mu \mathrm{M}$ EdU into each well for 2 hours. Then, cells were fixed with $4 \%$ polyformaldehyde at room temperature for 15 mins and subsequently incubated with Apollo staining solution for 30 mins. The cell nuclei were stained with Hoechst 33342 (1:1000 in PBS). The proportion of EdU-positive cells was determined with a Zeiss LSM780 confocal microscope system (Carl Zeiss, Germany). Image J_v1.8.0 (National Institutes of Health, USA) was used to count the number of all cells and proliferating cells.

\section{Irradiation}

Cells were ionize-irradiated (3.5 Gy/min) at room temperature using X-RAD225 OptiMAX Biological Irradiator (Precision X-Ray Inc., USA). Once irradiated, the cells were immediately transferred to the incubator at $37^{\circ} \mathrm{C}$ in $5 \% \mathrm{CO}_{2}$ until the designated harvest time.

\section{Immunofluorescent staining}

Cells grown on coverslips were fixed in $4 \%$ paraformaldehyde for $15 \mathrm{~min}$ at room temperature and then permeabilized with $0.1 \%$ Triton X-100 in PBS for 15 min. The cells were blocked with $5 \%$ goat serum for 30 min before incubation with primary antibodies overnight at $4^{\circ} \mathrm{C}$. After washing with PBS, cells were incubated with the secondary antibodies for $1 \mathrm{~h}$ at room temperature. The cell nuclei were dyed with Hoechst 33342 (1:1000 in PBS). After a final wash with PBS, the slides were covered with anti-fade mounting medium (Beyotime, China). Microscopic analyses were captured at magnification $(200 x, 630 x)$ with a Carl Zeiss LSM 780.

\section{Flow cytometry}

For cell apoptosis analysis, $1 \times 10^{6}$ cells were washed twice with PBS and stained in $100 \mu \mathrm{L}$ binding buffer with $5 \mu \mathrm{L}$ Annexin V-APC and $10 \mu \mathrm{L}$ 7-ADD for 20 mins in the dark at room temperature. Annexin V/PI 
staining assays were performed following the manufacturer's protocol (BD Biosciences, San Jose, CA, USA), Then, an additional $400 \mu \mathrm{L}$ of binding buffer was added to the cell suspension and the samples were determined by CytoFLEX S flow cytometry (Beckman, USA).

\section{Western blotting}

Cell lysates were extracted using RIPA lysis buffer (Beyotime Biotechnology, China) with protease inhibitor cocktail (APExBIO, China) and phosphatase cocktail (APExBIO, China). The protein concentration was measured by a BCA protein assay kit. The proteins were mixed with $5 \times$ loading buffer (EpiZyme, China) and boiled at $95^{\circ} \mathrm{C}$ for $5 \mathrm{~min}$ followed by separate in $10 \%$ SDS-PAGE (EpiZyme, China). The protein bands were electrophoretically transferred onto polyvinylidene difluoride membranes (PVDF, Roche, USA). The PVDF membranes were blocked with 5\% BSA for $1 \mathrm{~h}$ at room temperature and incubated overnight with primary antibodies in 1\% BSA. After incubation with secondary antibody at a dilution of 1:10000 for $1 \mathrm{~h}$ at room temperature, protein bands were visualized by Odyssey infrared imaging system ( $\mathrm{Li}$ CorBioscences, USA).

\section{Statistical analysis}

All quantitative data are represented as mean values of at least 3 independent experiments \pm standard deviation (SD). Multiple hypothesis testing based on FDR using the Perseus has been used to analyze the foldchange between cancer with para-cancerous tissues. Differences between groups were analyzed by Student's t-test for two groups and one-way ANOVA for more than two groups. Survival analysis was performed using Kaplan-Meier method and compared with the log-rank test. Pearson chi-squared test and Fisher's exact test were used to analyze the relationship between pSer290-BCLAF1 expression and clinical features. Cox proportional hazard model was used to determine factors related to patient survival. $\mathrm{P}<$ 0.05 was considered as statistically significant value. All statistical analyses were performed with GraphPad Prism 8.0.2 software.

\section{Results}

\section{Upregulation of DNA damage response in gastric cancer}

A modified label-free phospho-proteomic workflow was adapted to quickly identify possible dysregulated protein phosphorylation in GC tissues. Briefly, the shotgun proteomics was applied to the total proteins from tumor and para-cancerous tissues, and peptide sequences were repeatedly determined by deep fractionation and high-resolution liquid chromatography-tandem mass spectrometry (LC-MS/MS). By allowing single amino acid polymorphisms (SAAPs) in the peptides, the mass differences between the coding amino acids and actual residues were screened using the multi-blind spectral alignment algorithm MODa [25, 26] and Byonic [27]. Nonzero delta masses were clustered within intervals and mass clusters assessed by Gaussian regression to determine the potential protein modifications. Among them, 4243 delta masses were clustered as the well-defined phosphorylation modification (Fig. 1a, $+79.9665 \pm 0.0002$, $n=4243, R 2=0.91)$ that spread over 832 unique phosphorylated sites of 382 different proteins 
(Supplementary Table 1). In comparison of the cancer with para-cancerous tissues, 25 phosphorylated sites were up- and 52 down-regulated $(F C>2, p<0.05)$ (Fig. 1b). Notably, the upregulated protein phosphitylations were predominately involved in the interaction with nucleotides, DNAs, and RNAs (Fig. $1 \mathrm{c}, \mathrm{e})$, which were apparently associated with dysregulation of DDR, nuclear assembly and genomic instability of GC. Among them, a DDR-associated protein BCLAF1 is the mostly upregulated phosphoprotein. On the other hand, the mostly down-regulated protein phosphitylations were predominately involved in the regulation of muscle contraction, which reflected the function loss associated with GC (Fig. 1d, f). Consistently, GSEA unveiled that differentially expressed proteins (DEPs) in GC were involved in DNA replication $(p<0.0001)$ and cell cycle $(p<0.0001$, Fig. $1 \mathrm{~g})$. The functions of these DEPs were predominantly connected with the DNA damage repair pathways, including positive/negative regulation of DNA repair, DNA de-alkylation repair, mitochondrial DNA repair, DNA synthesis, and DNA ligation etc. (Fig. 1h). Taken together, our quick label-free phosphoproteomics suggest that BCLAF1 phosphorylation is upregulated and may be involved in the regulation of the DDR in GC.

\section{Upregulation of BCLAF1 Ser290 phosphorylation in gastric cancer}

Among the 7 phosphorylated residues we identified at BCLAF1, the most significantly upregulated site was Ser290 ( $F C=22.3, p=0.005, n=18)$ (Fig. 2a), locating at the THRAP3 domain of BCLAF1 involved in response to DNA damage [21]. To verify the phosphorylation at BCLAF1 Ser290 (pBCLAF1(Ser290)), total proteins were isolated from HGC-27 (HGC) GC cells and immunoprecipitated with anti-BCLAF1 antibody. The gel area with the positive signal was excised and digested with trypsin. The phosphorylated BCLAF1 peptide, YSPSQN(Sp290)PIHHIPSR, was confirmed by LC-MS/MS analysis (Fig. 2b). To further confirm the phosphorylation of BCLAF1 at Ser290, a synthetic peptide containing the phosphorylated Ser290 was used to generate a specific polyclonal antibody. Dot-blotting assay showed that the antibody specifically recognized the phosphorylated peptide (Fig. 2c). GFP-tagged wild-type BCLAF1 (WT), and BCLAF1 with Ser290A (S290A) or Ser290D (S290D) mutation were transiently overexpressed in HGC cells, the endogenous BCLAF1 and the GFP-tagged BCLAF1 (WT, S290A or S290D) were recognized by the antiBCLAF1 antibody. The anti-pBCLAF1(Ser290) antibody detected the endogenous pBCLAF1 and the GFPtagged WT pBCLAF1 (Fig. 2d); however, weak signal was detected in the unphosphorylated BCLAF1 with S290A/D mutants, confirming the specificity of the antibody on BCLAF1 Ser290 phosphorylation.

To explore the existence and clinical importance of pBCLAF1(Ser290) in human GC, the cancer tissue microarray chips were used to perform IHC analysis. pBCLAF1(Ser290) level was significantly upregulated in gastric tumor tissues compared to matched non-tumor tissues (Fig. 3a). The IHC staining scores were analyzed by the staining index. Statistically, the pBCLAF1(Ser290) level was found to be significantly higher in the gastric tumor tissues than that in adjacent non-tumor tissues (Fig. 3b), and the high level of pBCLAF1(Ser290) was positively correlated to poor prognosis (Fig. 3c). The correlation of pBCLAF1(Ser290) with clinicopathological characteristics in the tissue microarray is further summarized in Table 1, the high pBCLAF1(Ser290) level was found to be positively correlate with the age and stage of GC patients. Additionally, Univariate analysis revealed that pBCLAF1(Ser290) levels ( $p=0.021)$, Grade 
$(p=0.002), T$ stage $(p=0.002), N$ stage $(p<0.001)$ and TNM stage $(p<0.001)$ were significant prognostic factors for OS. Multivariate analysis further indicated Grade (HR: 1.691; 95\% Cl: 1.044-2.740; $p=0.033$ ), T stage (HR: $2.161 ; 95 \%$ Cl: 1.291-3.617; $p=0.003$ ) and $N$ stage (HR: $1.498 ; 95 \%$ Cl: $1.026-2.187 ; p=0.036)$ were shown to be available independent prognostic factors (Table 2). Together, these results confirmed the existence and upregulated level of pBCLAF1(Ser290) and suggested that the high pBCLAF1(Ser290) level is correlated to the GC development and poor prognosis in GC patients.

\section{BCLAF1 phosphorylation at serine 290 facilitates cell proliferation}

BCLAF1 has been reported to promote cell proliferation and invasion in hepatocellular carcinoma [19], indicating an oncogenic role of BCLAF1 in cancer. Thus, we first assessed the involvement of BCLAF1 in human GC. BCLAF1 deficient cell lines were established by transfecting lentiviral particles encoding shRNA hairpins against BCLAF1 or a nontargeting shRNA (shNC) into human GC cell lines HGC and AGS (Fig. 4a). MTT and colony formation assays indicated that BCLAF1 knockdown delayed cell growth significantly (Fig. 4b-d). Moreover, in vitro EdU incorporation assay showed BCLAF1 silent cells exhibited slower proliferation rate (Fig. 4e, f).

We then investigated if pBCLAF1(Ser290) affect the oncogenic function of BCLAF1 in GC cells. The cell viability was assessed in GC cells stably expressing WT, S290A, S290D types of BCLAF1 by MTT assay (Fig. 5a). The cell viability of cancer cells transfected with WT BCLAF1 was significantly higher than the cells with empty vector transfection. GC cells with BCLAF1 S290D demonstrated the highest viability at all time points compared with cells transfected with WT and S290A forms of BCLAF1. We then revaluated cell proliferation using colony formation assay and found that WT BCLAF1 enhanced the growth capability of HGC and AGS cells (Fig. 5b, c). Moreover, the number and size of colonies in the cells with BCLAF1 S290D were markedly higher and bigger than in the cells with WT and S290A forms of BCLAF1. Furthermore, using EdU incorporation assay, we found that WT BCLAF1 overexpression was associated with an increased mitotic rate compared to the empty vector cells (Fig. $5 \mathrm{~d}, \mathrm{e}$ ). The cell proliferation and mitotic rate in the cells with BCLAF1 S290D was markedly higher than in the cells with WT and S290A forms of BCLAF1. These data indicated that the oncogenic role of BCLAF1 is dependent, at least partially, on the phosphorylation of BCLAF1 at Ser290.

\section{BCLAF1 Ser290 phosphorylation is involved in DNA damage repair and promotes IR-induced DNA damage response}

It has been reported that BCLAF1 is a DDR-associated protein which usually form nuclear foci during DNA damage [23]. To determine whether BCLAF1 participates in the DNA damage repair in GC cells, we detected the expression and sub-cellular localization of BCLAF1 after ionizing radiation (IR) exposure. HGC cells were treated with X-rays at different doses and determined BCLAF1 expression by Immunofluorescence. The BCLAF1 and $\mathrm{y}-\mathrm{H} 2 \mathrm{AX}$ colocalized in nuclei and the levels of BCLAF1 and $\mathrm{y}^{-}$ $\mathrm{H} 2 \mathrm{AX}$ were increased dramatically after IR in a dose-dependent manner (Fig. 6a). As Y-H2AX connotes the existence and degree of DNA damages, and BCLAF1 is reported to colocalize with $\gamma-\mathrm{H} 2 \mathrm{AX}$ foci in nuclei and stabilize the Ku70/DNA-PKcs complex, facilitating non-homologous end joining (NHEJ)-based DNA 
damage repair [23], to gain an insight into a possible role for BCLAF1 in the DNA damage repair, $\mathrm{y}-\mathrm{H} 2 \mathrm{AX}$ foci formation following IR was analyzed by immunofluorescence staining. As shown in Fig. 6b, the $\mathrm{Y}^{-}$ H2AX foci formation was almost two-fold higher in BCLAF1 silent cells than in control cells after IR with a dose of $15 \mathrm{~Gy}$ at $2 \mathrm{~h}$. The results suggested that BCLAF1 is involved in DNA damage repair and BCLAF1 deficiency delays DNA damage repair and causes cells to maintain a higher level of DNA damage.

We then assessed the importance of Ser290 phosphorylation in the role of BCLAF1 in the DNA damage repair. Western blotting showed that pBCLAF1(Ser290) level increased after IR besides the increase of BCLAF1 protein level (Fig. 7a). In addition, pBCLAF1(Ser290) colocalized with $\mathrm{y}-\mathrm{H} 2 \mathrm{AX}$ upon irradiation and formed strong IR-induced foci following IR at a dose dependent (Fig. 7b). To further validated the effect of Ser290 phosphorylation in mediating the role of BCLAF1 in the DNA damage repair, HGC and AGS cells were transfected with S290 mutant forms of BCLAF1 (Fig. 7c). Indeed, the overexpression of the non-phosphorylatable S290A mutant led to a significant reduction of DNA damage repair upon IR as determined by the $\mathrm{Y}-\mathrm{H} 2 \mathrm{AX}$ foci formation. The S290D mutant decreased the radiosensitivity when compared to WT BCLAF1 overexpressing HGC and AGS cells. Our results collectively indicated that BCLAF1 Ser290 phosphorylation is responsible for the role of BCLAF1 in DNA damage repair.

\section{BCLAF1 phosphorylation at serine 290 protects cancer cells from IR-induced cell apoptosis}

Given that BCLAF1 is actively involved in DNA damage repair, the effect of BCLAF1 on IR-induced cell apoptosis was evaluated by flow cytometry analysis at $24 \mathrm{~h}$ after $15 \mathrm{~Gy}$ X-ray exposure (Fig. 8a). BCLAF1 knockdown cells exhibited significantly more apoptosis than the shNC group post-IR in AGS cells $(26.98 \pm 1.01 \%$ vs $17.85 \pm 0.47 \%, P<0.001)$. Similarly, the proportion of apoptotic cells was significantly induced compared with that in the shNC group post-IR in HGC cells $(7.15 \pm 0.39 \%$ vs $5.55 \pm 0.12 \%, P<0.05)$, indicating that BCLAF1 knockdown increases cell apoptosis induced by IR. Overall, we found that BCLAF1 knockdown cells showed a reduction in cell proliferation and an elevated basal $\mathrm{Y}-\mathrm{H} 2 \mathrm{AX}$ foci and higher susceptible to IR-induced DNA damages and cell apoptosis, suggesting that BCLAF1 expression might affect the radiosensitivity of GC cells.

We further validated the effect of Ser290 phosphorylation of BCLAF1 on IR-induced apoptosis. AGS and HGC cells that stably expressed WT, S290D, or S290A forms of BCLAF1 were treated with 15 Gy X-ray. We collected each group of cells for apoptosis analyses (Fig. 8b). AGS and HGC cells with WT BCLAF1 overexpression were more resistant to IR than empty vector cells. Moreover, we found that substitution of Ser290 with A or D led to the promotion or inhibition of apoptosis in AGS and HGC cells post-IR. In conclusion, these results indicated that the phospho-deficiency of BCLAF1 Ser290 enhances IR-induced DNA damage and apoptosis, suggesting that the Ser290 phosphorylation is responsible for the function of BCLAF1 on the radiosensitivity of GC cells.

\section{Discussion}

In the present study, a modified label-free phospho-proteomic workflow was adapted to systematically identify dysregulated protein phosphorylation in GC. 832 unique phosphorylated sites spreading over 382 
proteins were identified. In comparison with para-cancerous tissues, the phosphorylation of Ser290 at BCLAF1, a DNA damage response-associated protein, is the mostly upregulated in GC tissues.

BCLAF1 was a widely expressed gene that encodes a protein with homology to the basic zipper and Myb DNA binding domains [28]. Studies have indicated that BCLAF1 involves in diverse biological processes, such as DNA damage repair, cell proliferation and angiogenesis [14-16, 23]. Consistent with these reports, our results indicated that BCLAF1 knockdown reduced cell proliferation and increased cell susceptible to IR-induced DNA damages in GC cells. Recent studies indicated that BCLAF1 function mainly depends on its phosphorylation, for example, Ewald Heroes et al. reported that the BCLAF1:SDS22 interaction is dependent on the phosphorylation of BCLAF1 [29]. By searching the uniport protein database, 46 phosphorylation sites were found in BCLAF1, but the significance and function of these sites have not been reported. In this manuscript, we firstly found that level of pBCLAF1(Ser290) in GC tissues is significantly increased and can be used to predict poor survival outcome for GC patients, indicating that pBCLAF1(Ser290) may play an important role in GC. Therefore, clarifying how pBCLAF1(Ser290) regulates $\mathrm{GC}$ has important implications for understanding the pathogenesis and progression of GC. In GC cells, we showed that pBCLAF1(Ser290) was increased upon IR expose and promoted DNA damage repair and protected cells from IR-induced cell apoptosis. Thus, our results indicated that Ser290 phosphorylation is one of the critical forms of BCLAF1 modification in regulation its function in GC.

The protein $\mathrm{H} 2 \mathrm{AX}$ is rapidly phosphorylated at the serine 139 site $(\mathrm{\gamma}-\mathrm{H} 2 \mathrm{AX})$ in response to extensive DNA damage [30]. $\mathrm{Y}-\mathrm{H} 2 \mathrm{AX}$ induction is one of the earliest events in DNA damage response and plays an important role in the perception and repair of DNA damage [31] by promoting stable accumulation of many other signaling and DNA repair proteins including 53BP1 [32], GADD45A [33] and BRCA1 [34] at DSB sites. Particularly, BCLAF1 is an IR-induced H2AX-interacting partner involved in $\mathrm{Y}$-H2AX-mediated regulation of DNA damage repair and cell apoptosis [23]. Our data confirmed that IR induced the phosphorylation and co-localization of BCLAF1 and y-H2AX. Moreover, mimic and deficient Ser290 phosphorylation in BCLAF1 significantly affected the DDR and IR-induced apoptosis. These indicated the significance of Ser290 phosphorylation in the involvement of BCLAF1 in DDR and subsequent cancer cell apoptosis. However, the underlying mechanisms of how IR increase the pBCLAF1(Ser290) and how this phosphorylated BCLAF1 is recruited to the DNA damage loci need further investigation.

\section{Conclusions}

Taken together, we reported a serial of GC-associated phosphoproteins, further identification and analysis of these protein modifications will expand our understanding of GC. The identification of the upregulation of pBCLAF1(Ser290) suggested a potential biomarker for the prognosis of patients and a possible target to improve radiotherapy sensitivity in GC.

\section{Abbreviations}


BCA: Bicinchoninic Acid; BCLAF1: Bcl-2-associated transcription factor 1; BSA: bovine serum albumin; DDR: DNA damage response; DMEM: Dulbecco's modified Eagle's medium; EdU: 5-ethynyl-2》deoxyuridine; FBS: fetal bovine serum; FDR: false discovery rate; GC: Gastric cancer; $\gamma-\mathrm{H} 2 \mathrm{AX}$ : phosphohistone H2AX; HPLC: high performance liquid chromatography; IAA: iodoacetamide; IR: ionizing radiation; LC-MS/MS: label-free liquid chromatography-tandem mass spectrometry; OD: optical density; PTM: Posttranslational modification; PVDF: polyvinylidene difluoride membranes; SAAPs: single amino acid polymorphisms; SD: standard deviation; Ser290: serine 290; TFA: trifluoroacetic acid; TMA: tissue microarray; UA: urea

\section{Declarations}

\section{Acknowledgments}

We thank Dr. Z. Yan for coordinating the distribution of tissue samples and discussions on data analysis.

\section{Authors' contributions}

GW, J-HY and JL designed the research; JL, JL, ZS, YD, FW and ZY performed the experiments and analyzed the data; JL wrote the manuscript. J-HY directed proteomics experiments, guided the bioinformatics analysis, and wrote the manuscript. GW conceived the study, designed and oversaw the study, evaluated data, and wrote the manuscript. All authors read and approved the manuscript.

\section{Funding}

This study was supported by grants to G.W. from the National Key R\&D Program of China (2017YFC1308600), National Natural Science Foundation of China (81872148), Natural Science Foundation of Shandong Province (ZR2019MH002), and J.-H.Y. from National Natural Science Foundation of China (32070157) and Tai-Shan Scholarship with Xinfa Pharmaceutical of Shandong Province.

\section{Availability of data and materials}

The datasets used and/or analysed during the current study are available from the corresponding author on reasonable request.

\section{Ethics approval and consent to participate}

The present study was approved by the Ethics Committee and Institutional Review Board of Shandong University

\section{Consent for publication}

Not applicable. 


\section{Competing Interests}

The authors have declared that no competing interest exists

\section{References}

1. Busuttil RA, George J, House CM, Lade S, Mitchell C, Di Costanzo NS, et al. SFRP4 drives invasion in gastric cancer and is an early predictor of recurrence. Gastric Cancer. 2020.

2. Hu K, Wang S, Wang Z, Li L, Huang Z, Yu W, et al. Clinicopathological risk factors for gastric cancer: a retrospective cohort study in China. BMJ Open. 2019;9(9):e030639.

3. Min BH, Kim KM, Park CK, Lee JH, Rhee PL, Rhee JC, et al. Outcomes of endoscopic submucosal dissection for differentiated-type early gastric cancer with histological heterogeneity. Gastric Cancer. 2015;18(3):618-26.

4. Lee J, Chung SJ, Choi JM, Han YM, Kim JS. Clinicopathologic Characteristics and Long-Term Outcome of Gastric Cancer Patients with Family History: Seven-Year Follow-Up Study for Korean Health Check-Up Subjects. Gastroenterol Res Pract. 2020;2020:4028136.

5. Qiao YQ, Zheng L, Jia B, Wang WH, Zheng XH, Fan LL, et al. Risk factors for surgical-site infections after radical gastrectomy for gastric cancer: a study in China. Chin Med $\mathrm{J}$ (Engl). 2020;133(13):1540-5.

6. Ashraf N, Hoffe S, Kim R. Adjuvant treatment for gastric cancer: chemotherapy versus radiation. Oncologist. 2013;18(9):1013-21.

7. Sawyer TK, Shakespeare WC, Wang Y, Sundaramoorthi R, Huang WS, Metcalf CA 3. Protein phosphorylation and signal transduction modulation: chemistry perspectives for small-molecule drug discovery. Med Chem. 2005;1(3):293-319. rd, et al.

8. Medvedev AE, Piao W, Shoenfelt J, Rhee SH, Chen H, Basu S, et al. Role of TLR4 tyrosine phosphorylation in signal transduction and endotoxin tolerance. J Biol Chem. 2007;282(22):1604253.

9. Proud CG. Phosphorylation and Signal Transduction Pathways in Translational Control. Cold Spring Harb Perspect Biol. 2019;11(7).

10. Kuznetsova KG, Solovyeva EM, Kuzikov AV, Gorshkov MV, Moshkovskii SA. [Modification of cysteine residues for mass spectrometry-based proteomic analysis: facts and artifacts]. Biomed Khim. 2020;66(1):18-29.

11. Lao YW, Gungormusler-Yilmaz M, Shuvo S, Verbeke T, Spicer V, Krokhin OV. Chromatographic behavior of peptides containing oxidized methionine residues in proteomic LC-MS experiments: Complex tale of a simple modification. J Proteomics. 2015;125:131-9.

12. Chouchani ET, James AM, Fearnley IM, Lilley KS, Murphy MP. Proteomic approaches to the characterization of protein thiol modification. Curr Opin Chem Biol. 2011;15(1):120-8. 
13. Yan GR, Ding W, Xu SH, Xu Z, Xiao CL, Yin XF, et al. Characterization of phosphoproteins in gastric cancer secretome. OMICS. 2011;15(1-2):83-90.

14. Mou SJ, Yang PF, Liu YP, Xu N, Jiang WW, Yue WJ. BCLAF1 promotes cell proliferation, invasion and drug-resistance though targeting IncRNA NEAT1 in hepatocellular carcinoma. Life Sci. 2020;242:117177.

15. Wen Y, Zhou X, Lu M, He M, Tian Y, Liu L, et al. Bclaf1 promotes angiogenesis by regulating HIF1alpha transcription in hepatocellular carcinoma. Oncogene. 2019;38(11):1845-59.

16. Zhou X, Li X, Cheng Y, Wu W, Xie Z, Xi Q, et al. BCLAF1 and its splicing regulator SRSF10 regulate the tumorigenic potential of colon cancer cells. Nat Commun. 2014;5:4581.

17. Scherr AL, Gdynia G, Salou M, Radhakrishnan P, Duglova K, Heller A, et al. Bcl-xL is an oncogenic driver in colorectal cancer. Cell Death Dis. 2016;7(8):e2342.

18. Jiang T, Liu B, Wu D, Zhang F. BCLAF1 induces cisplatin resistance in lung cancer cells. Oncol Lett. 2020;20(5):227.

19. Shao AW, Sun H, Geng Y, Peng Q, Wang P, Chen J, et al. Bclaf1 is an important NF-kappaB signaling transducer and C/EBPbeta regulator in DNA damage-induced senescence. Cell Death Differ. 2016;23(5):865-75.

20. Li X, He Z, Cheng B, Fang Q, Ma D, Lu T, et al. Effect of BCLAF1 on HDAC inhibitor LMK-235-mediated apoptosis of diffuse large $B$ cell lymphoma cells and its mechanism. Cancer Biol Ther. 2018;19(9):825-34.

21. Vohhodina J, Barros EM, Savage AL, Liberante FG, Manti L, Bankhead P, et al. The RNA processing factors THRAP3 and BCLAF1 promote the DNA damage response through selective mRNA splicing and nuclear export. Nucleic Acids Res. 2017;45(22):12816-33.

22. Soodgupta D, White LS, Yang W, Johnston R, Andrews JM, Kohyama M, et al. RAG-Mediated DNA Breaks Attenuate PU.1 Activity in Early B Cells through Activation of a SPIC-BCLAF1 Complex. Cell Rep. 2019;29(4):829-43. e5.

23. Lee $Y Y, Y u$ YB, Gunawardena HP, Xie L, Chen X. BCLAF1 is a radiation-induced H2AX-interacting partner involved in gammaH2AX-mediated regulation of apoptosis and DNA repair. Cell Death Dis. 2012;3:e359.

24. Liu H, Lu ZG, Miki Y, Yoshida K. Protein kinase $C$ delta induces transcription of the TP53 tumor suppressor gene by controlling death-promoting factor Btf in the apoptotic response to DNA damage. Mol Cell Biol. 2007;27(24):8480-91.

25. Na S, Bandeira N, Paek E. Fast multi-blind modification search through tandem mass spectrometry. Mol Cell Proteomics. 2012;11, M111.010199.

26. Na S, Kim J, Paek E, MODplus. Robust and Unrestrictive Identification of Post-Translational Modifications Using Mass Spectrometry. Analytical chemistry. 2019;91:11324-33.

27. Bern M, Kil YJ, Becker C. Byonic: advanced peptide and protein identification software. Curr Protoc Bioinformatics. 2012;Chap. 13, Unit13 20. 
28. Kasof GM, Goyal L, White E. Btf, a novel death-promoting transcriptional repressor that interacts with Bcl-2-related proteins. Mol Cell Biol. 1999;19(6):4390-404.

29. Heroes E, Van der Hoeven G, Choy MS, Garcia JDP, Ferreira M, Nys M, et al. Structure-Guided Exploration of SDS22 Interactions with Protein Phosphatase PP1 and the Splicing Factor BCLAF1. Structure. 2019;27(3):507-18. e5.

30. Rogakou EP, Pilch DR, Orr AH, Ivanova VS, Bonner WM. DNA double-stranded breaks induce histone H2AX phosphorylation on serine 139. J Biol Chem. 1998;273(10):5858-68.

31. Zhao H, Huang X, Halicka HD, Darzynkiewicz Z. Detection of Histone H2AX Phosphorylation on Ser139 as an Indicator of DNA Damage. Curr Protoc Cytom. 2019;89(1):e55.

32. Djuzenova CS, Elsner I, Katzer A, Worschech E, Distel LV, Flentje M, et al. Radiosensitivity in breast cancer assessed by the histone gamma-H2AX and 53BP1 foci. Radiat Oncol. 2013;8:98.

33. Liu J, Jiang G, Mao P, Zhang J, Zhang L, Liu L, et al. Down-regulation of GADD45A enhances chemosensitivity in melanoma. Sci Rep. 2018;8(1):4111.

34. Papadaki C, Sfakianaki M, loannidis G, Lagoudaki E, Trypaki M, Tryfonidis K, et al. ERCC1 and BRAC1 mRNA expression levels in the primary tumor could predict the effectiveness of the second-line cisplatin-based chemotherapy in pretreated patients with metastatic non-small cell lung cancer. $J$ Thorac Oncol. 2012;7(4):663-71.

\section{Tables}

Table 1. Correlation between pBCLAF1(Ser290) expression and clinicopathological characteristics 


\begin{tabular}{|c|c|c|c|c|c|c|}
\hline & \multirow{2}{*}{ variables } & \multicolumn{2}{|c|}{ pBCLAF1(Ser290) expression } & \multirow{2}{*}{ total } & \multirow[t]{2}{*}{$x^{2}$} & \multirow[t]{2}{*}{$\mathrm{p}$ value } \\
\hline & & low & High & & & \\
\hline \multirow[t]{3}{*}{ Age (year) } & & & & & 4.357 & 0.037 \\
\hline & $<60$ & 11 & 14 & 25 & & \\
\hline & $>=60$ & 14 & 50 & 64 & & \\
\hline \multirow[t]{3}{*}{ T stage } & & & & & 0.189 & 0.664 \\
\hline & $\mathrm{T} 1 / \mathrm{T} 2$ & 4 & 8 & 12 & & \\
\hline & $\mathrm{T} 3 / \mathrm{T} 4$ & 21 & 56 & 77 & & \\
\hline \multirow[t]{3}{*}{ TNM stage } & & & & & 0.714 & 0.398 \\
\hline & $I / I I$ & 11 & 22 & 33 & & \\
\hline & III/IV & 14 & 42 & 56 & & \\
\hline \multirow[t]{3}{*}{$\mathrm{N}$ stage } & & & & & 4.445 & 0.035 \\
\hline & NO & 9 & 10 & 19 & & \\
\hline & N1/N2/N3 & 16 & 54 & 70 & & \\
\hline \multirow[t]{3}{*}{ M stage } & & & & & 2.287 & 0.130 \\
\hline & Mo & 23 & 63 & 86 & & \\
\hline & M1 & 2 & 1 & 3 & & \\
\hline \multirow[t]{3}{*}{ Sex } & & & & & 1.420 & 0.233 \\
\hline & Female & 4 & 18 & 22 & & \\
\hline & Male & 21 & 46 & 67 & & \\
\hline \multirow[t]{3}{*}{ grade } & & & & & 0.084 & 0.771 \\
\hline & $I / I I$ & 7 & 16 & 23 & & \\
\hline & III/IV & 18 & 48 & 66 & & \\
\hline
\end{tabular}

Table 2. Univariate and multivariate analyses of the factors correlated with Overall survival of Gastric carcinoma patients. 


\begin{tabular}{|llllllll|}
\hline variables & \multicolumn{2}{l}{ Univariate analysis } & \multicolumn{4}{c|}{ Multivariate analysis } \\
& HR & $\mathbf{9 5 \%} \mathrm{Cl}$ & $\mathbf{p ~ v a l u e}$ & HR & $\mathbf{9 5 \%} \mathrm{Cl}$ & p value \\
\hline expression & 1.935 & $1.106-3.386$ & 0.021 & 1.684 & $0.958-2.962$ & 0.070 \\
\hline sex & 0.696 & $0.410-1.183$ & 0.181 & & & \\
\hline Grade & 2.135 & $1.317-3.462$ & 0.002 & 1.691 & $1.044-2.740$ & 0.033 \\
\hline Age & 1.016 & $0.993-1.040$ & 0.166 & & & \\
\hline T stage & 1.805 & $1.232-2.645$ & 0.002 & 2.161 & $1.291-3.617$ & 0.003 \\
\hline N stage & 1.484 & $1.199-2.837$ & 0.000 & 1.498 & $1.026-2.187$ & 0.036 \\
\hline M stage & 1.594 & $0.499-5.093$ & 0.431 & & & \\
\hline TNM stage & 2.001 & $1.377-2.908$ & 0.000 & 0.878 & $0.418-1.843$ & 0.731 \\
\hline
\end{tabular}

Figures 
a

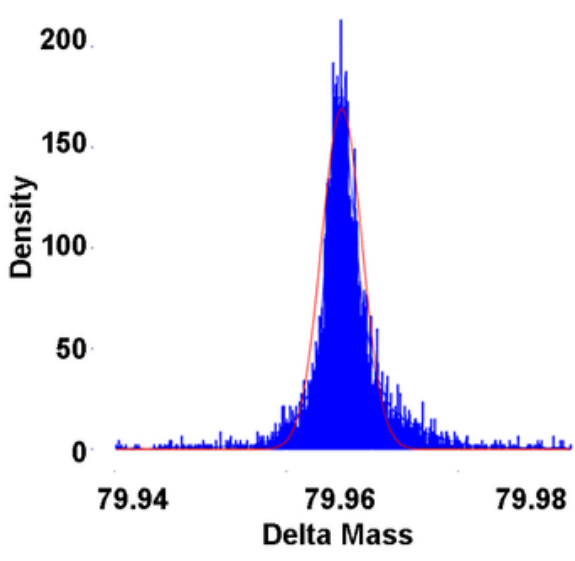

d

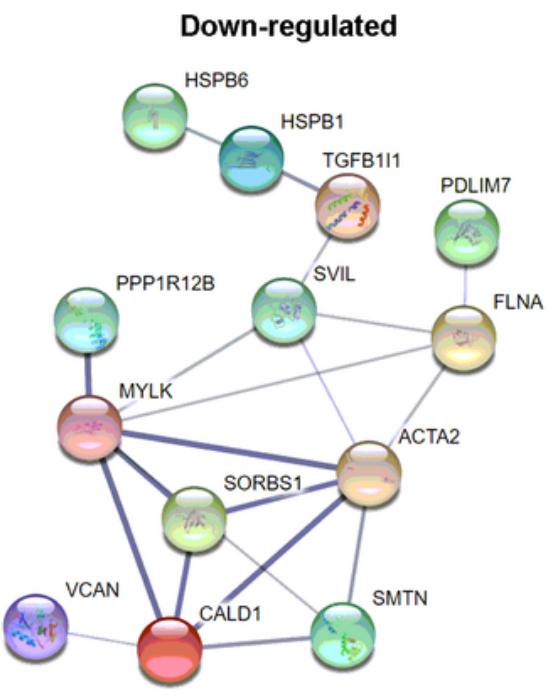

g

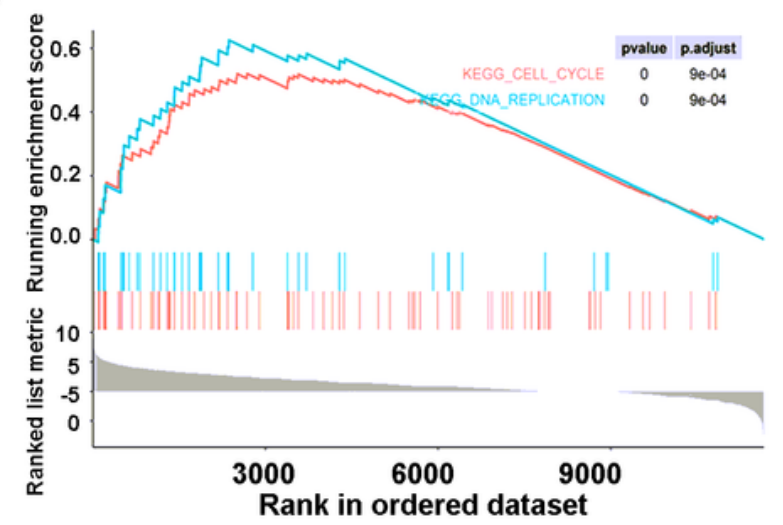

b

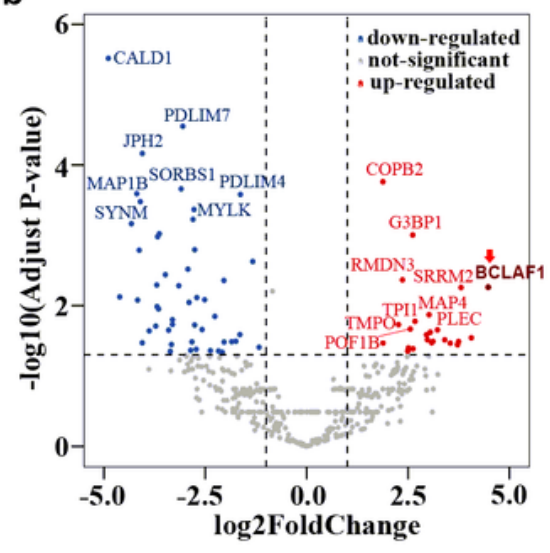

e

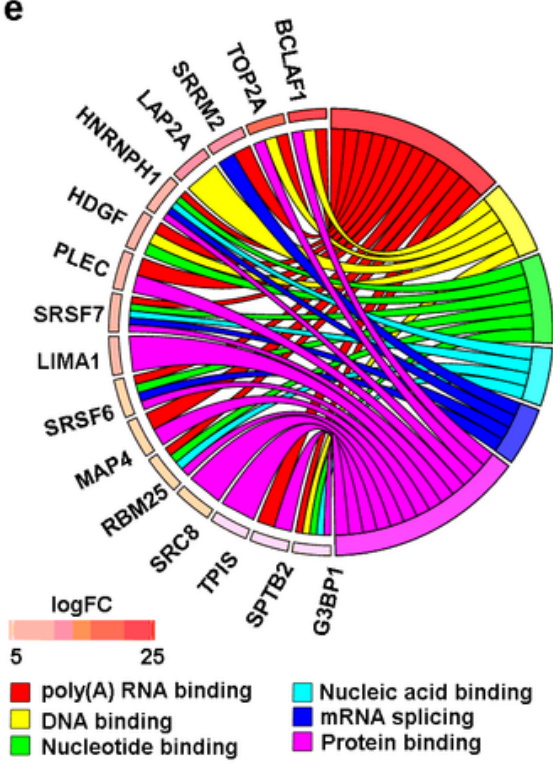

C

Up-regulated

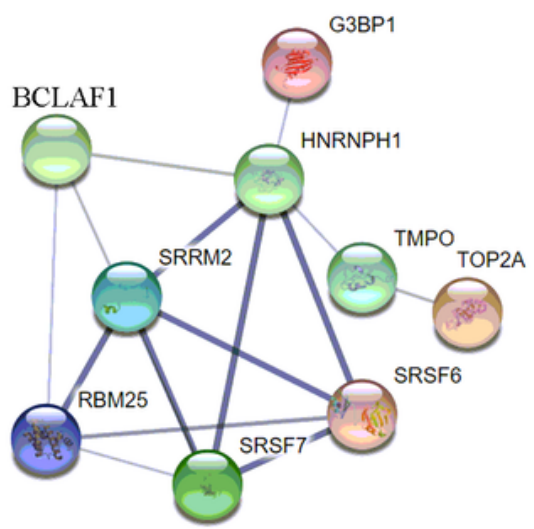

f

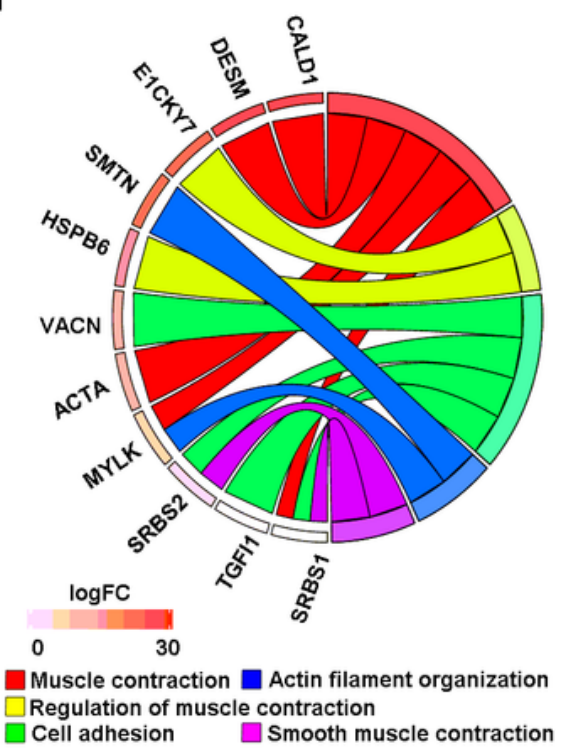

h

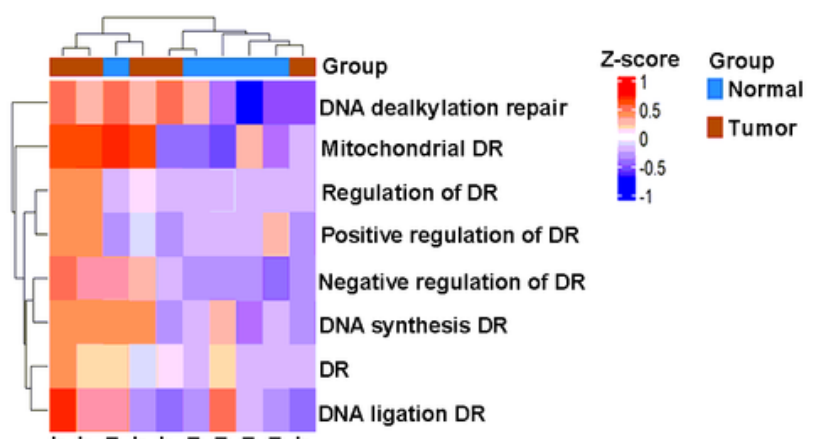

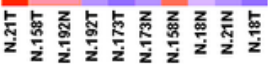

\section{Figure 1}

Dysregulated DNA damage response in gastric cancer unraveled by quick label-free phosphoproteomics.

(a) Phosphopeptide ratio distribution. (b) Volcano plot to show the differential phosphoproteins with unique phospho-sites in gastric cancer and paired non-tumor tissues, with candidate phosphoproteins marked, including BCLAF1 (dark red). The red points represented the up-regulated phosphoproteins, while blue points represented the down-regulated phosphoproteins. Multiple hypothesis testing based on FDR. 
(c) PPI of up-regulated phosphoproteins. (d) PPI of down-regulated phosphoproteins. (e) The chord diagram of enrichment analysis for the up-regulated phosphoproteins. (f) The chord diagram of enrichment analysis for the down-regulated phosphoproteins. (g) GSEA analysis of the DEPs between gastric cancer and paired non-tumor tissues. (h) GSVA was used to analyze these DEPs closely related to DNA repair.

a

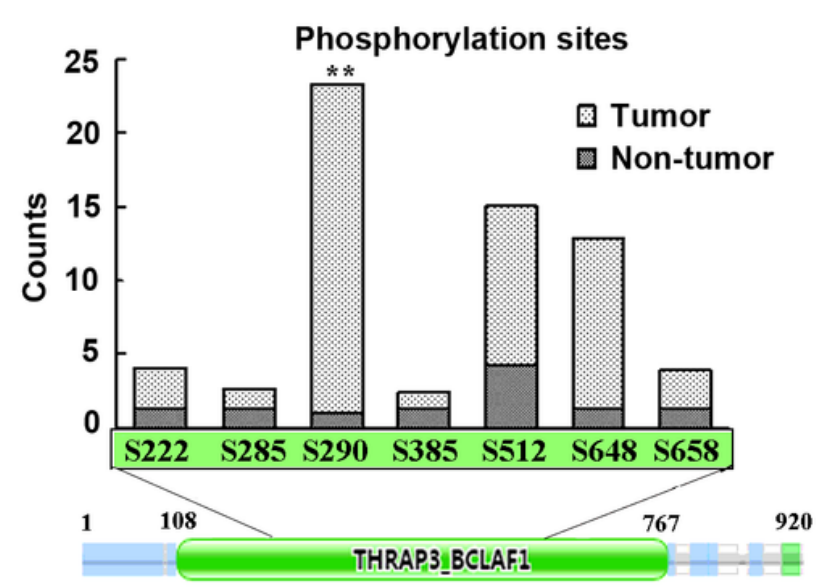

b

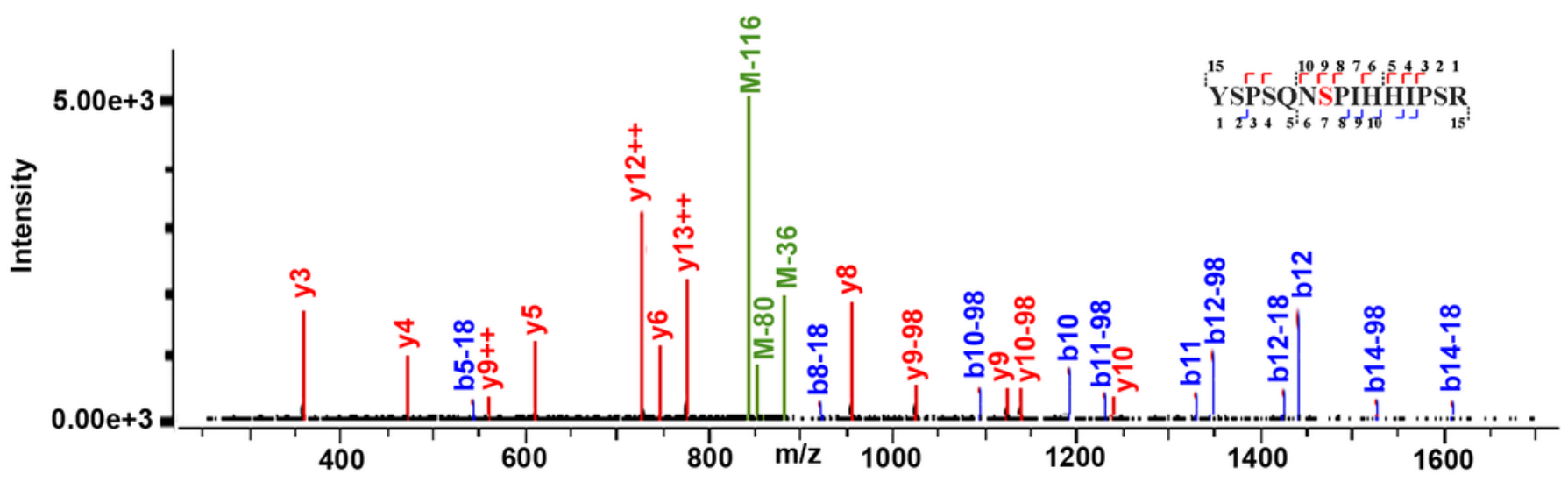

c

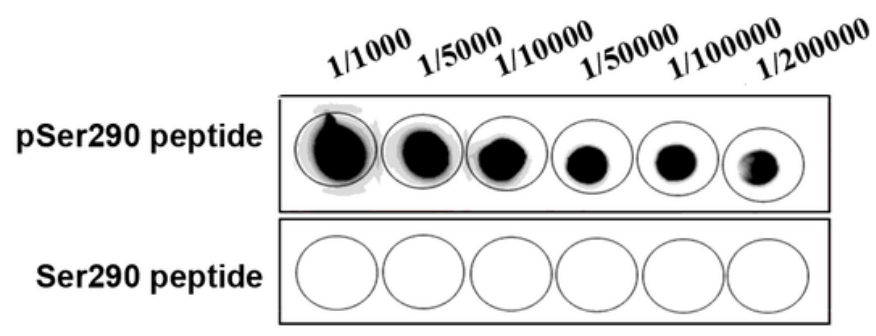

d

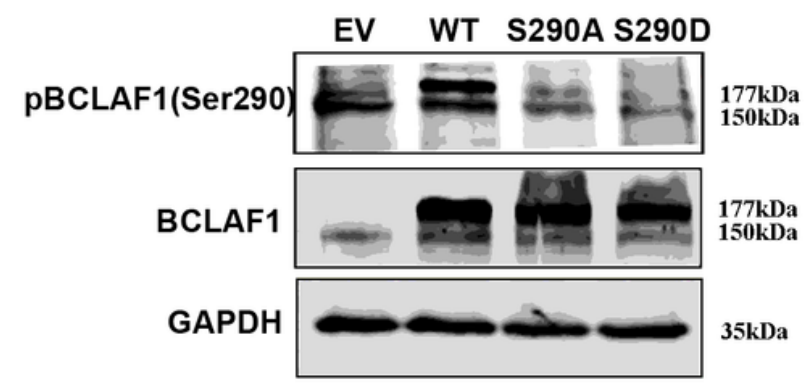

Figure 2

Phosphorylation of BCLAF1 Ser290 is significantly increased in gastric cancer. (a) Total counts of phosphorylated residues in BCLAF1. ${ }^{*}$ p $<0.01$ vs. Non-tumor based on Student's t-test. (b) MS/MS spectrum of pSer290-containing peptide, YSPSQN(pS290)PIHHIPSR in HGC gastric cancer cells. (c) Dot blotting of the phosphorylated (pSer290) and unmodified (Ser290) peptides with anti-pBCLAF1(Ser290) antibody. (d) Western blotting of the total proteins from HGC cells overexpressing WT, S290A and S290D forms of BCLAF. 177 kD, GFP-tagged BCLAF; 150 kD, endogenous BCLAF. 
a
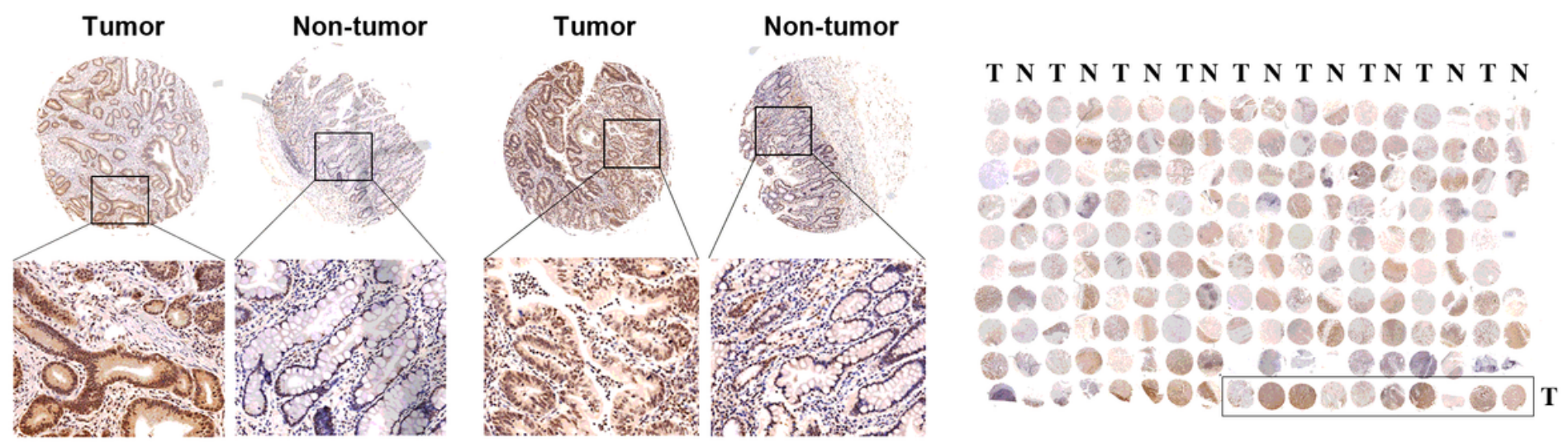

b

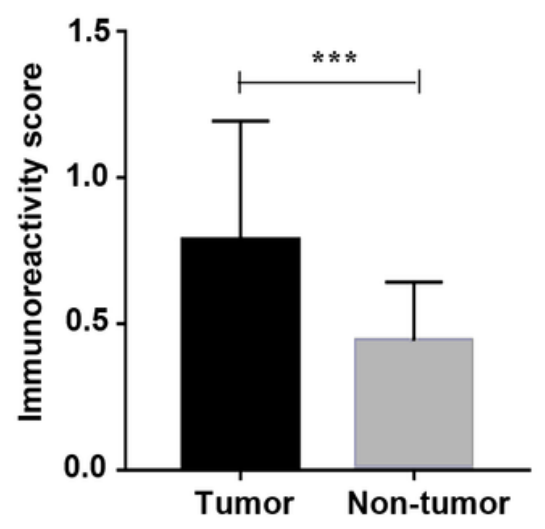

c

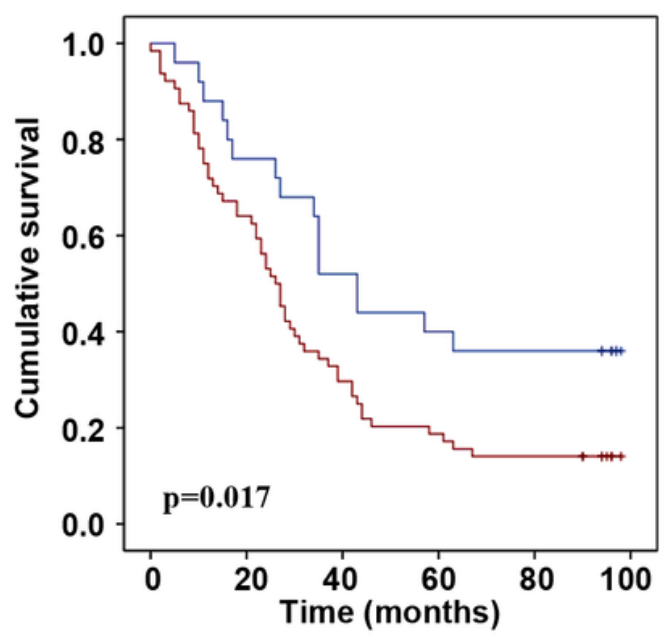

pBCLAF1(Ser290) expression $\rightarrow$ Low

$\rightarrow$ High

+ Low-censored

+ High-censored

\section{Figure 3}

BCLAF1 Ser290 phosphorylation is correlated with gastric cancer development. (a) Immunohistochemistry on gastric tumor tissues and adjacent non-tumor tissues using pBCLAF1(Ser290) antibody (up-panel 40x and lower panel 100x, respectively). (b) Statistical data to show the IHC score of pBCLAF1(Ser290) staining in the gastric tumor tissues $(n=95)$ and adjacent non-tumor tissues $(n=85)$. $\star * \star p<0.001$, Student's t-test. (c) Kaplan-Meier survival curve showed that high pBCLAF1(Ser290) level correlated with poor overall survival of GC patients. $p=0.017$, log-rank test. 
a

HGC

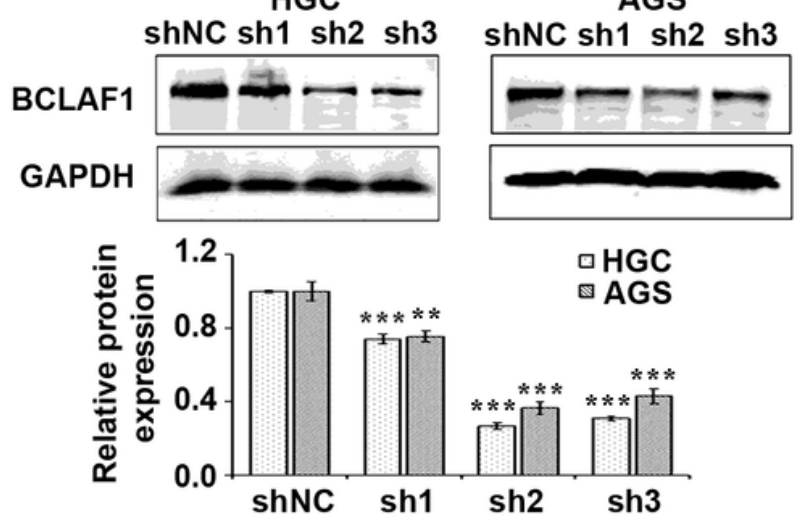

C

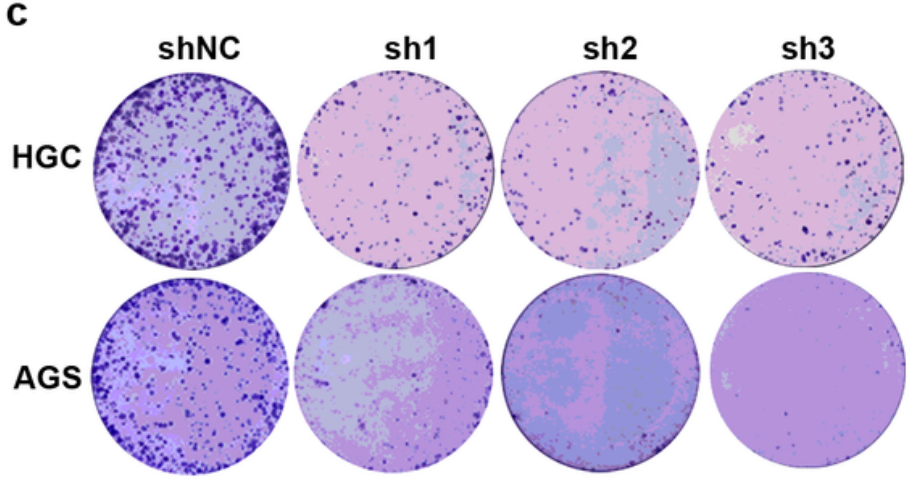

b
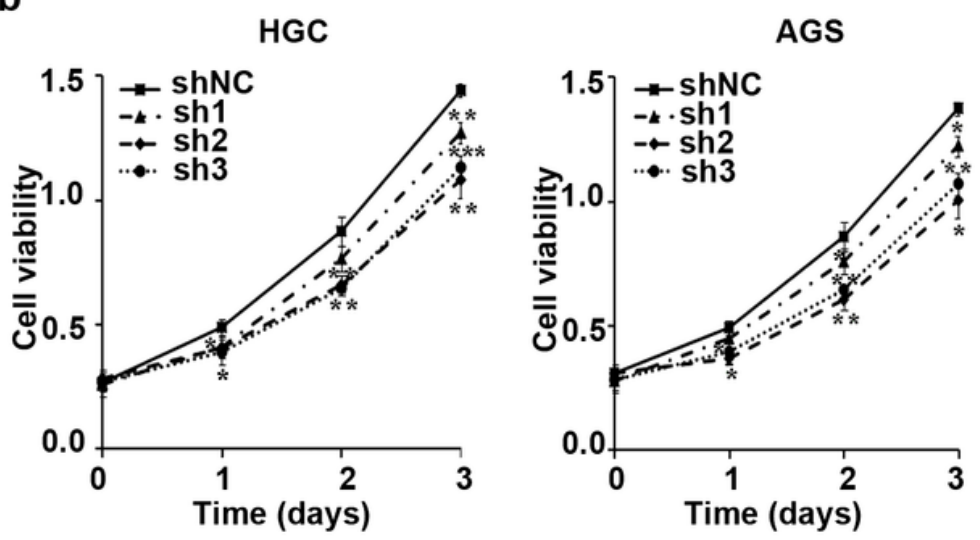

d

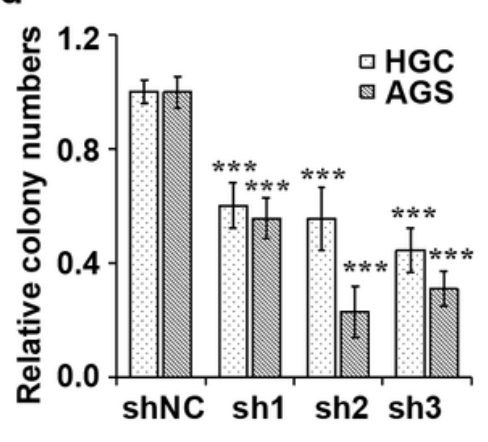

f

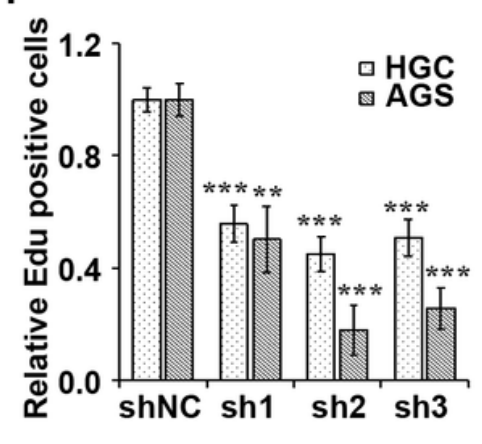

e

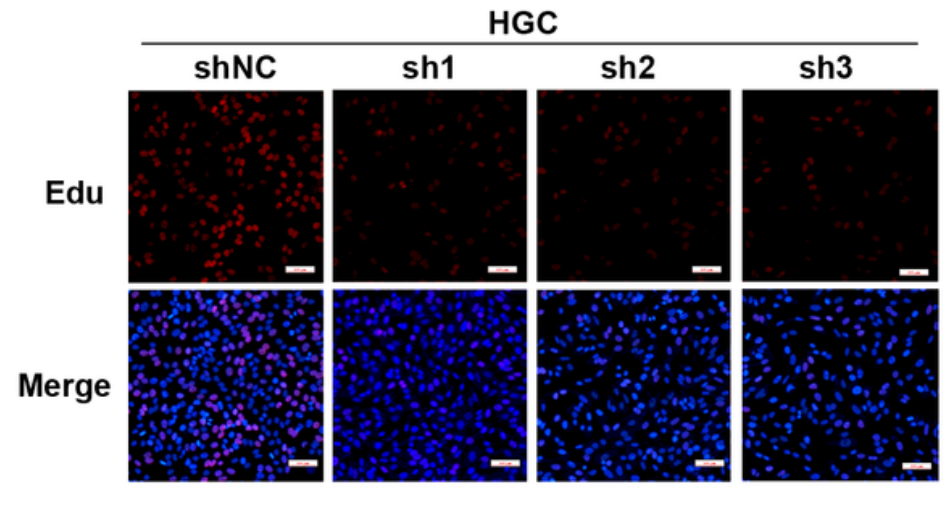

AGS

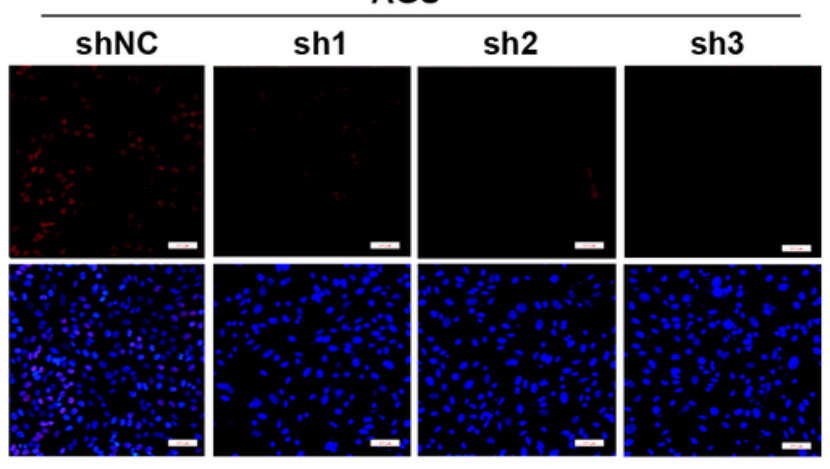

Figure 4

BCLAF1 silence inhibits cell proliferation. (a) Western blot analysis of BCLAF1 expression in HGC and AGS cells infected with shNC or shBCLAF1s (sh1, sh2 and sh3). GAPDH served as an internal reference. (b) The transfected cells were harvested for MTT assay. BCLAF1 depletion inhibited cell proliferation of HGC and AGS gastric cancer cells. (c) Colony formation assay was performed to investigate colony formation ability of shNC or shBCLAF1s cells. (d) Quantitative results of colony formation analyzed with Image J. (e) EdU assay was used to examine the cell proliferation ability of shBCLAF1s transfected cells. Scale bars, $100 \mu \mathrm{m}$. (f) Three different fields were randomly chosen and quantitative results of EdU assay analyzed with Image J. Data presented as the mean \pm SD of three independent experiments. ${ }^{*} p<0.05$, ${ }^{* *} \mathrm{p}<0.01$ and ${ }^{* * *} \mathrm{p}<0.001$ vs. shNC based on Student's t-test. 
a

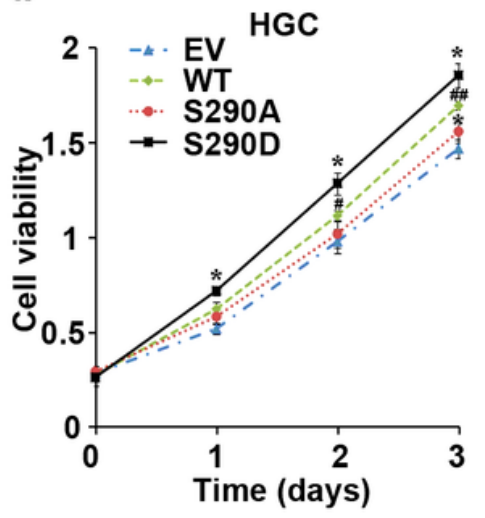

d
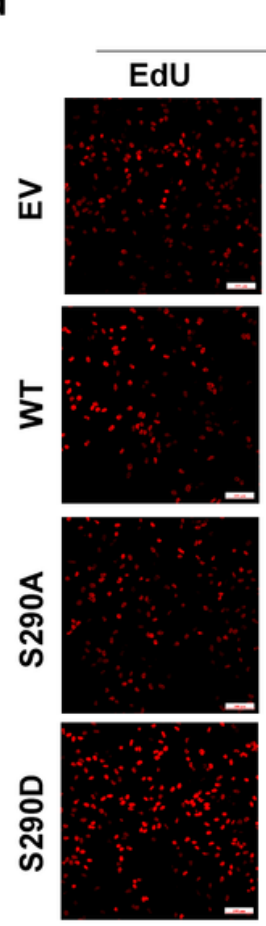

HGC
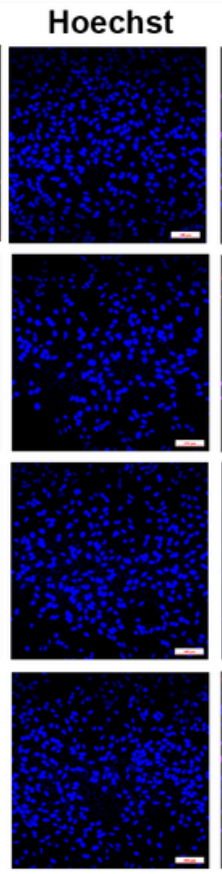

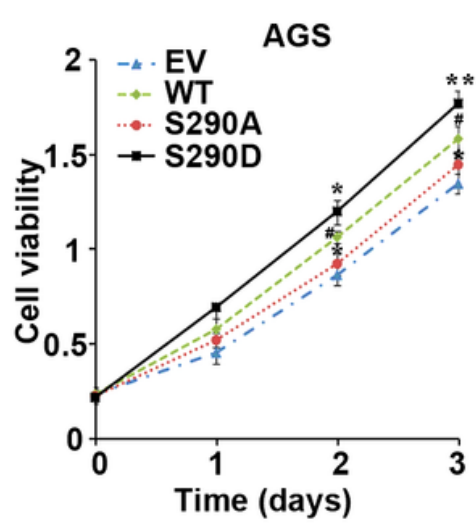

b
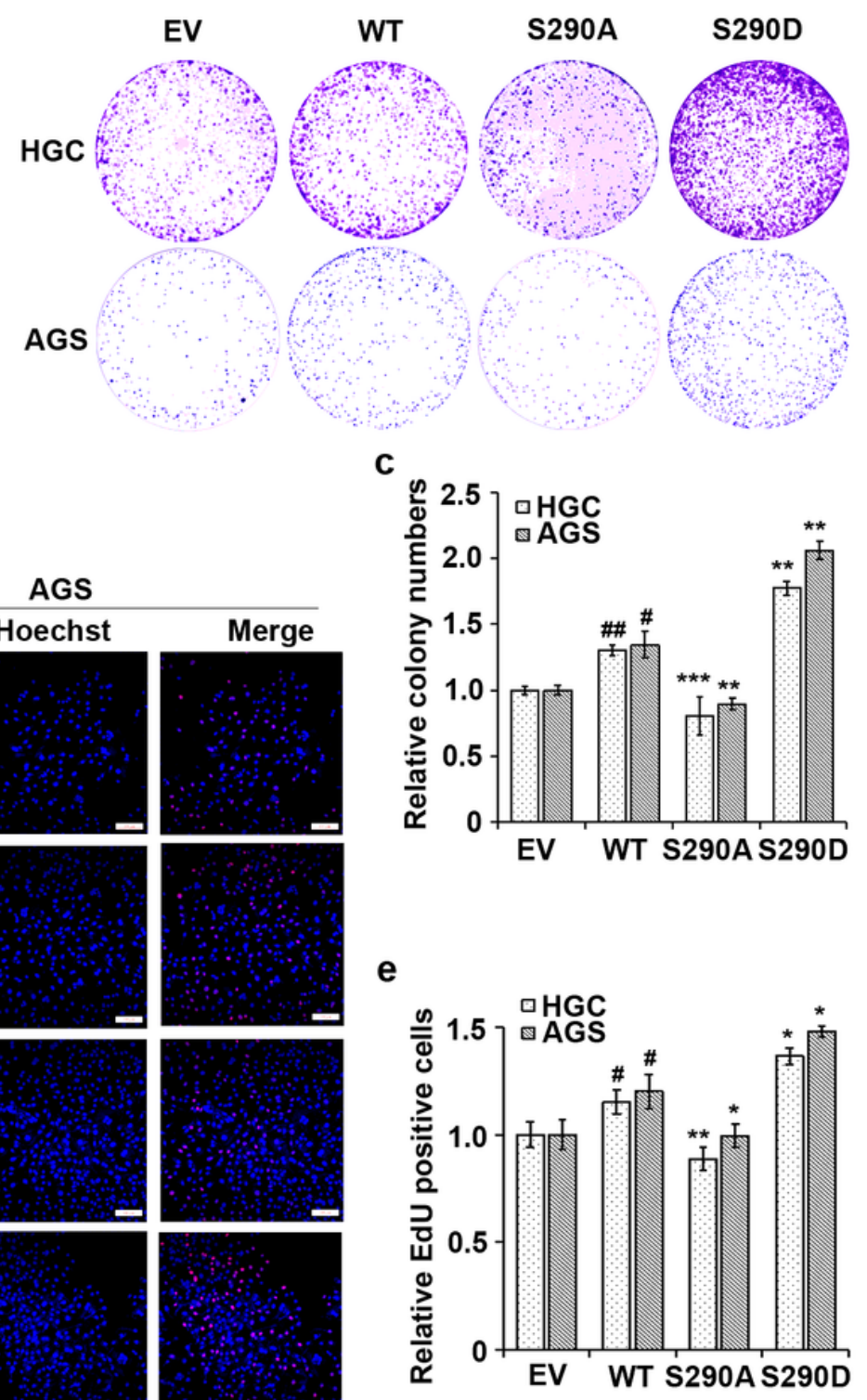

Figure 5

BCLAF1 phosphorylation at Ser290 facilitates cell proliferation. (a) MTT assay of HGC and AGS cells overexpressing BCLAF1-WT (WT), mimic BCLAF1-pSer290 (S290D), nonphosphorylatable Ser290 (S290A) or empty vector (EV). (b) Colony formation assay was performed to investigate colony formation ability in HGC and AGS cells overexpressing WT, S290D, S290A forms of BCLAF1. (c) Quantitative results of colony formation analyzed with Image J. (d) EdU incorporation assay was performed using a fluorescence method in cells transfected with WT, S290D, S290A forms of BCLAF1. Scale bars, $100 \mu \mathrm{m}$.

(e) For each group, three different fields were randomly chosen and EdU positive cells were counted with Image J. Data are shown as mean \pm SD. ${ }^{*} p<0.05$, ${ }^{* *} p<0.01$ and ${ }^{* * *} p<0.001$ vs. WT. \#p $<0.05, \# \# p<$ 0.01 vs. EV based on Student's t-test. 


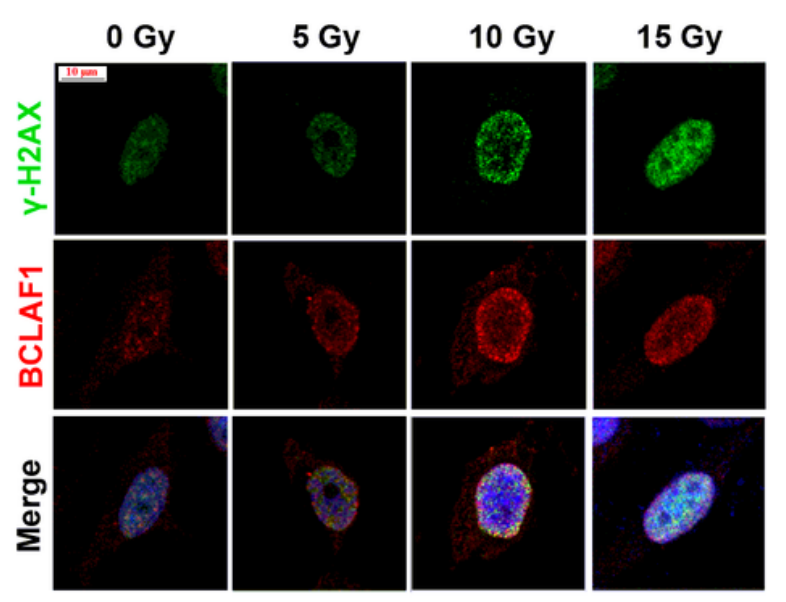

b

HGC

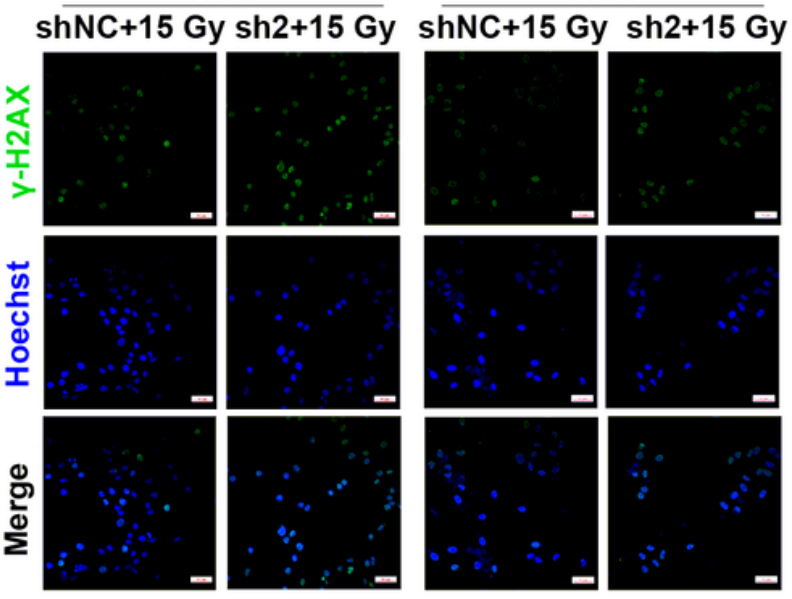

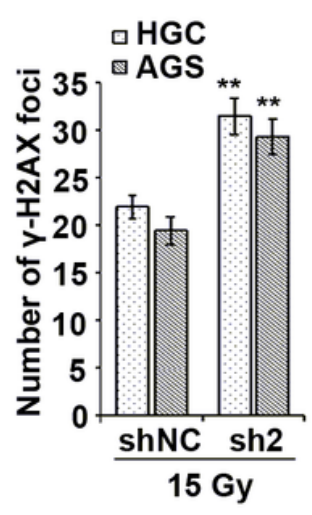

Figure 6

BCLAF1 phosphorylation at Ser290 is involved in DNA damage repair. (a) HGC cells were fixed and stained with indicated antibodies at 2 hours after different doses irradiation (0, 5, 10 or 15 Gy). (b) The shNC and sh2 stable cells were treated with IR (15 Gy for 2 hour), $y-H 2 A X$ level was determined using immunofluorescence. Scale bars, $100 \mu \mathrm{m}$. Number of $\mathrm{Y}$-H2AX foci per cell were quantified and statistically analyzed by Student's t-test. ${ }^{\star \star} p<0.01$ vs. shNC+15 Gy. 
a

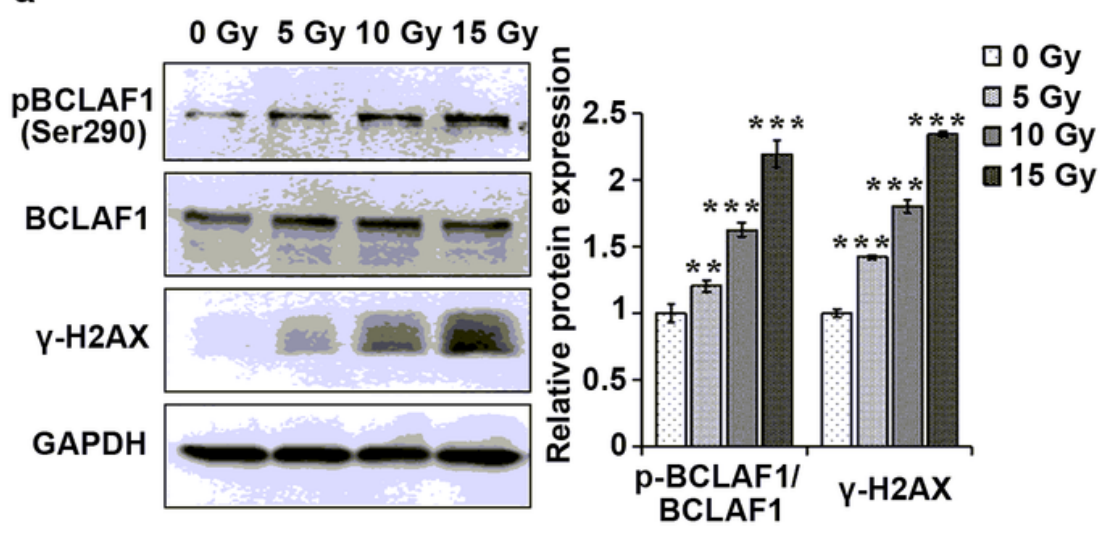

C

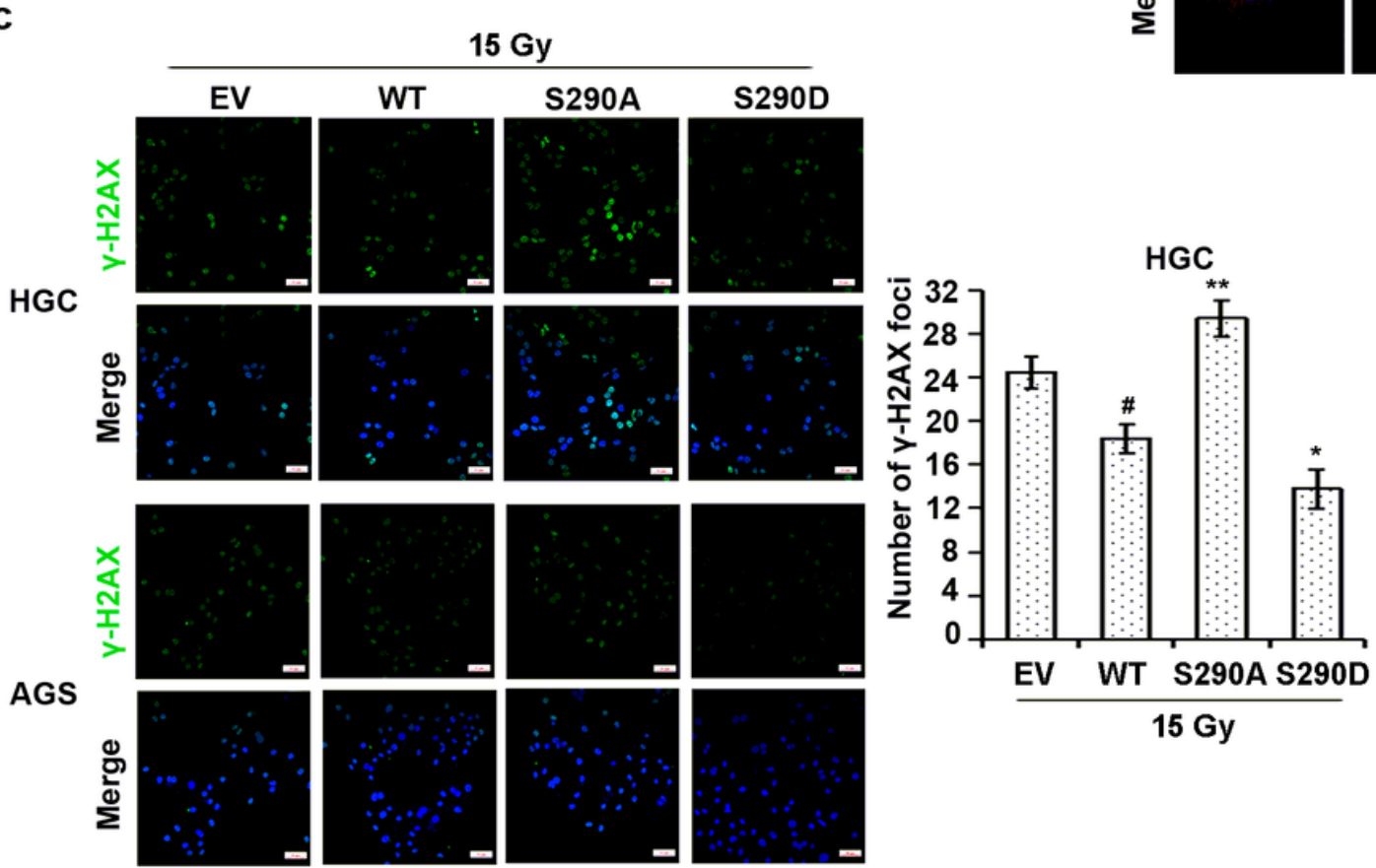

b

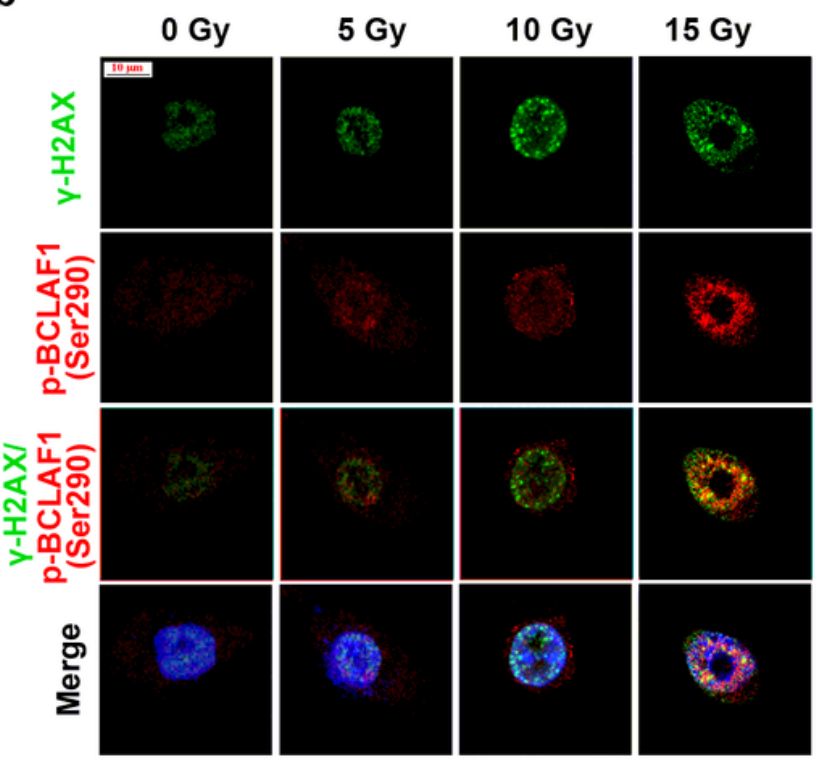

\section{Figure 7}

BCLAF1 phosphorylation at Ser290 is involved in DNA damage repair and promotes IR-induced DNA damage responses. (a) HGC cells were treated with 0, 5, 10 or 15 Gy IR for 2 hours. Western blot was performed to detect pBCLAF1(Ser290) and BCLAF1. ${ }^{*} p<0.05,{ }^{*} p<0.01$ and ${ }^{\star * *} p<0.001$ vs. 0 Gy. (b) HGC cells were exposed to IR $(0,5,10$ or $15 \mathrm{~Gy})$ for 2 hours. Cells were double-stained with monoclonal antibody to $\mathrm{Y}-\mathrm{H} 2 \mathrm{AX}$ and polyclonal antibody to pBCLAF1(Ser290). IR-induced pBCLAF1(Ser290) foci were colocalized with $\mathrm{Y}-\mathrm{H} 2 \mathrm{AX}$ foci. Scale bars, $100 \mu \mathrm{m}$. (c) HGC and AGS stably cells overexpressing WT, S290D, S290A forms of BCLAF1 were treated with IR (15 Gy for 2 hour), $y-H 2 A X$ level was determined using immunofluorescence. Scale bars, $100 \mu \mathrm{m}$. Numbers of $\mathrm{Y}-\mathrm{H} 2 \mathrm{AX}$ foci per cell were quantified and statistically analyzed by Student's t-test. ${ }^{*} p<0.05$ and ${ }^{* *} p<0.01$ vs. WT+15 Gy, \#p $<0.05$ vs. EV+15 Gy. 

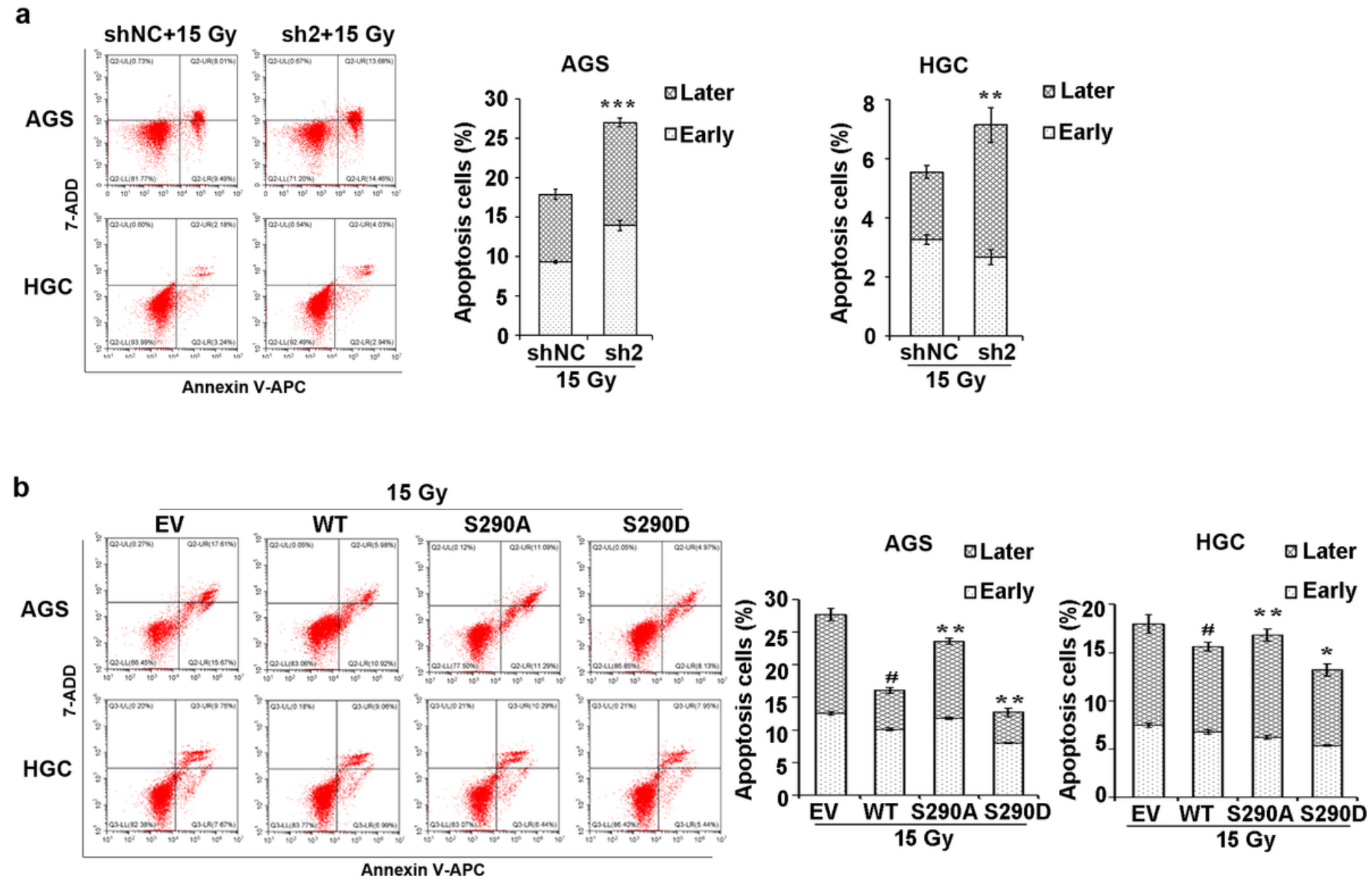

Figure 8

BCLAF1 phosphorylation at Ser290 protects cells from IR-induced cell apoptosis. (a) The shNC and sh2 stable cells were treated with 15 Gy IR for 2 hours, and the percentage of cell apoptosis was determined using flow cytometry. Data are shown as mean \pm SD of three independent experiments. ${ }^{*} p<0.05, * \star p<$ 0.01 and $* * * p<0.001$ vs. shNC+15 Gy. (b) Moreover, HGC and AGS cells stably overexpressing WT, S290D, S290A forms of BCLAF1 were treated with 15 Gy IR for 2 hours, and the percentage of cell apoptosis was determined using flow cytometry. Data are shown as mean \pm SD of three independent experiments. ${ }^{*} p<0.05$ and ${ }^{*} p p<0.01$ vs. WT+15 Gy, \#p $<0.05$ vs. EV+15 Gy based on Student's t-test. 